AleXandra N. LenZ

\title{
Zur variationslinguistischen Analyse regionalsprachlicher Korpora ${ }^{1}$
}

\begin{abstract}
Im Beitrag werden korpuslinguistische Aspekte und Fragestellungen aus variationslinguistischer Perspektive diskutiert, wobei der Fokus auf der Analyse regionalsprachlicher Daten und Korpora des Deutschen liegt. Unter Regionalsprache wird hier der areal gebundene, sprechsprachliche Gesamtbereich „unterhalb" der normierten Standardsprache verstanden, der sich von den lokalen Basisdialekten über Varietäten bzw. Sprechlagen des mittleren Bereichs bis hin zum standardnächsten Pol der Regionalsprache (Regionalakzent oder Regionalstandard) erstreckt.

Am Beginn des Beitrags steht ein Überblick über Datenklassen, die als empirische Grundlage für regionalsprachliche Fragestellungen herangezogen werden können. Eine Präsentation regionalsprachlicher Korpora, die auf den vorgestellten Datenklassen aufbauen, schließt sich an, wobei hier lediglich einige ausgewählte, online zugängliche Korpora Berücksichtigung finden (können). In einem nächsten Schritt werden die vorgestellten Korpora zur konkreten variationslinguistischen Analyse von zwei regionalsprachlichen Phänomenen aus dem lautlichen (,g-Spirantisierung“) bzw. grammatischen Bereich (,Rezipientenpassiv“) herangezogen und auf ihre Ergiebigkeit geprüft.
\end{abstract}

\section{Zu Spezifik und Problematik regionalsprachlicher Daten und Korpora}

Im vorliegenden Beitrag werden korpuslinguistische Aspekte und Fragestellungen aus variationslinguistischer Perspektive diskutiert. Dabei wird der Fokus auf der Analyse regionalsprachlicher Daten und Korpora liegen.

Regionalsprache wird hier definiert als der areal gebundene, sprechsprachliche Gesamtbereich „unterhalb“ der normierten Standardsprache. ${ }^{2}$ Dieser variative Gesamtbereich erstreckt sich von den lokalen Basisdialekten über Varietäten bzw. Sprechlagen des mittleren Bereichs bis hin zum standard-

I Für kritische Anmerkungen und hilfreiche Literaturhinweise danke ich herzlich Stephan Elspaß (Augsburg), Joachim Herrgen (Marburg), Arend Mihm (Duisburg) und Jürgen Erich Schmidt (Marburg).

2 Hinter den Attributen „unten“ versus „hoch“ steht das Bild von der Zweidimensionalität der Regionalsprache. In diesem Bild entspricht die $\mathrm{x}$-Achse der horizontal-arealen Ebene, während die y-Achse die vertikal-soziale Dimension von Sprache repräsentiert. Zum Begriff der Zweidimensionalität s. etwa Bellmann 1983 und Auer/Hinskens 1996. 
nächsten Pol der Regionalsprache, dem sogenannten „Regionalakzent“ (Lenz 2003) oder „Regionalstandard“ (Spiekermann 2005). ${ }^{3}$

Aus der dem Regionalsprachenbegriff inhärenten Oralität und Variabilität leiten sich spezielle korpuslinguistische Probleme ab. Zu diesen gehören etwa das im Zusammenhang mit der Gewinnung oraler Daten häufig diskutierte „Beobachterparadoxon", das nach William Labov (1972, S. 61 f.) darin besteht, dass die Erhebung natürlichen Sprachverhaltens eigentlich impliziert, Sprachverhalten gerade dann zu beobachten, wenn Sprecher nicht beobachtet werden. ${ }^{4}$ Mit wachsendem Methodenbewusstsein gehen in der Variationslinguistik ausgefeilte Erhebungsmethoden einher, mit denen eine Minimierung der Auswirkungen des Paradoxons und die Gewinnung möglichst „authentischer" Sprachdaten angestrebt werden. Häufig bedingen diese Erhebungsmethoden eine Zunahme des personellen, zeitlichen, technischen und damit auch finanziellen Aufwands. Hinsichtlich der Erhebung natürlicher Sprachdaten kommt erschwerend hinzu, dass

"the low frequency of occurence in normal speech of any specific sound, word, grammatical item, pragmatic pattern, or whatever else is being sought, means that a very large amount of natural language must be observed or collected in order to have a sufficient quantity for useful analysis."

(Spolsky 2005, S. 1086)

Auch die Datenstrukturierung mittels Meta-Beschreibungen ${ }^{5}$ erweist sich als aufwändiges Moment (z. B. die areale oder soziale Einordnung von Sprachdaten, die ethnographische und soziodemographische Charakterisierung der Sprecher u. a.). Sind orale Sprachdaten erhoben und strukturiert, stellt die zeitintensive Datenaufbereitung in Form von (möglicherweise feinphonetischen) Transkriptionen und weiterführenden linguistischen Annotationen ein weiteres Problem dar, das sich aus der Spezifik gesprochensprachlicher „Primärdaten" 6 ergibt. Bei der Entscheidung für ein konkretes Transkriptionssystem bzw. für eine bestimmte „Tiefe“ der Transkription spielen

$3 \mathrm{Zu}$ den komplexen Gesamtstrukturen der modernen deutschen Regionalsprachen ist bislang relativ wenig bekannt. Als gesichert gilt, dass im Vergleich der Großräume des deutschsprachigen Sprachraums von der Schweiz bis hin zu Norddeutschland mit deutlich differierenden Verhältnissen zu rechnen ist. Zur Varietätenstruktur, die etwa für den moselfränkischen Raum und darüber hinaus wohl für weite Teile des Westmitteldeutschen anzusetzen ist, s. Lenz 2003. Zu regional differierenden Strukturmodellen im Deutschen s. die Überblicksdarstellungen bei Schmidt 1998, König $\left({ }^{15} 2005\right.$, S. 135 f.) und Spiekermann 2005.

4 Zum Beobachterparadoxon s. auch Wodak 1982 und Thun 2005.

s Nach Skiba (2005, S. 1189) fasst eine Meta-Beschreibung ,alle soziolinguistisch und technisch relevanten Informationen, die zu einer session [abgeschlossenen Dateneinheit; A. N. L.] gehören, zusammen und kann deshalb zum Aufbau eines gut beschriebenen Sprachkorpus verwendet werden."

6 Skiba (2005, S. 1187): „Audio- und Videoaufnahmen von sprachlichen Ereignissen bilden die primären Daten, und somit die Grundlage soziolinguistischer Untersuchungen." 
Aspekte der angestrebten Datenauswertung eine Rolle. ${ }^{7} \mathrm{Zu}$ diesen gehören Fragestellungen wie: Welche linguistische Systemebene steht im Fokus der Analyse? Welche Varianten bzw. welche Grade der Variantendifferenzierung sind bei der Analyse des in Rede stehenden Phänomens zu berücksichtigen? Welche sprachlichen Varianten konstituieren eine regionalsprachliche Variable?

Der Beitrag, in dem diese und andere korpuslinguistische Probleme der Regionalsprachenforschung diskutiert werden, gliedert sich wie folgt: Am Beginn steht ein Überblick über Datenklassen, die als empirische Grundlage für regionalsprachliche Fragestellungen herangezogen werden können (Kap. 2). Eine Präsentation regionalsprachlicher Korpora, die auf den vorgestellten Datenklassen aufbauen, schließt sich an (Kap. 3), wobei hier lediglich einige ausgewählte, online zugängliche Korpora Berücksichtigung finden (können). In einem nächsten Schritt werden die vorgestellten Korpora zur konkreten Analyse von zwei Beispielphänomenen herangezogen und auf ihre Ergiebigkeit geprüft (Kap. 4). Der Beitrag schließt mit einer Zusammenfassung (Kap. 5).

\section{Regionalsprachliche Datenklassen}

Ein Blick in die Forschungslandschaft zeigt, dass zur Analyse regionalsprachlicher Phänomene bzw. zum Aufbau regionalsprachlicher Korpora unterschiedlichste Daten herangezogen werden. Neben „objektiven“ Sprachdaten rücken zunehmend „subjektive" Spracheinstellungsdaten in den Untersuchungsfokus. ${ }^{8}$ Die Tatsache, dass Spracheinstellungsdaten in der Variationslinguistik nicht nur als Erklärungsfaktoren für Sprachverhalten herangezogen werden, sondern mitunter einen eigenständigen Untersuchungsgegenstand darstellen ${ }^{9}$, kommt in der Klassifikation regionalsprachlicher Daten in Abbildung (1) darin zum Ausdruck, dass auf einer ersten Klassifikationsebene Spracheinstellungsdaten neben Sprachdaten gestellt werden. Dabei wird das enge Verhältnis der beiden Datenkomplexe, die im Optimalfall miteinander erhoben und aufeinander beziehbar werden, durch den bidirektionalen Pfeil angedeutet.

7 Zur Diskussion verschiedener Transkriptionssysteme und -konventionen s. Richter 2005.

8 Nach Stickel (1999, S. 17) ,spricht man in der deutschsprachigen Linguistik seit Anfang der 70er Jahre von ,Spracheinstellungen' und meint damit wertende Dispositionen, die einzelne Menschen oder soziale Gruppen gegenüber sprachlichen Erscheinungen haben. Spracheinstellungen sind besonders Haltungen gegenüber Sprachen, Sprachvarietäten oder Sprachverhalten anderer Individuen oder Gruppen, oft mit wertender Berücksichtigung der jeweils eigenen Sprache. Wie andere Einstellungen gelten Spracheinstellungen als erlernt, relativ beständig, wenn auch veränderbar."

9 Zur Diskussion s. Lenz (2003, S. 263-265), Tophinke/Ziegler (im Druck) und Häcki Buhofer 2002. Als Beispiel für eine regionalsprachliche Studie, deren Schwerpunkt auf Einstellungsdaten liegt, sei hier Huesmann 1998 genannt. 
Der Fokus wird im Folgenden auf dem Komplex der Sprachdaten liegen. Diese werden auf einer nächsten Klassifizierungsstufe in medial mündliche und medial schriftliche Daten eingeteilt. Dabei bezieht sich - in Anlehnung an das Konzept von Koch/Oesterreicher (1985 und 1994) $-{ }^{10}$ das Medium auf die „Realisierung sprachlicher Äußerungen, wo ,mündlich“ = ,phonisch' und ,schriftlich“ = ,graphisch' ist" (Koch/Oesterreicher 1994, S. 587). Dagegen trägt der Konzeptionsbegriff der Tatsache Rechnung, dass innerhalb ein und desselben Mediums verschiedene Grade an raum-zeitlicher, sozialer und emotionaler Nähe bzw. Distanz und damit verbunden verschiedene Grade der sprachlichen Variation auftreten können. Bezüglich des Mediums sind die Begriffe ,mündlich\%,schriftlich' dichotomisch, bezüglich der Konzeption von Sprache und Sprechen stellen sie Endpunkte eines Kontinuums dar. Wie Koch/Oesterreicher (1994, S. 587) hervorheben, besteht zwar eine „prinzipielle Unabhängigkeit von Medium und Konzeption“, die jedoch „nicht im Widerspruch dazu [steht], daß einerseits zwischen dem phonischen Medium und konzeptionell mündlichen Äußerungsformen, andererseits zwischen dem graphischen Medium und konzeptionell schriftlichen Äußerungsformen eine ausgeprägte Affinität besteht.“

\section{(1) Regionalsprachliche Datenklassen (ein Überblick) ${ }^{11}$}

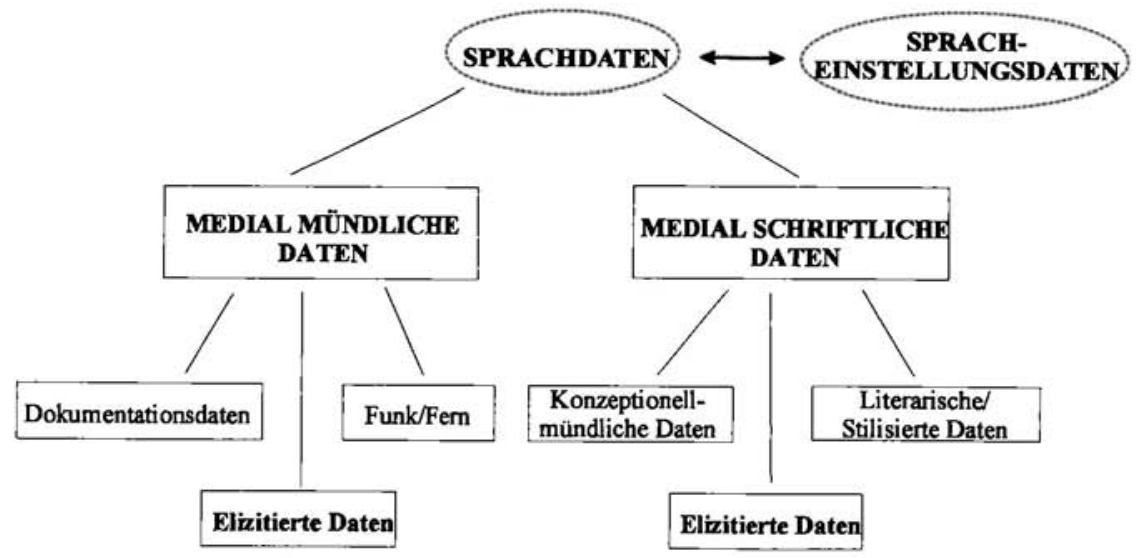

Abb. 1

Die in Abbildung (1) aufgeführten literarischen bzw. stilisierten Daten medial schriftlichen Typs stellen einen (bislang) eher vernachlässigten Datentyp in der Regionalsprachenforschung dar. ${ }^{12}$ Sie stehen in Konkurrenz mit konzep-

10 Eine Weiterentwicklung des Ansatzes liefern Ágel/Hennig 2006.

$11 \mathrm{Zu}$ einer Typologie von Sprachkorpora s. vergleichend Lemnitzer/Zinsmeister (2006, S. 102-107).

12 Zur Spezifik und Problematik von „Dialekt als Sprache literarischer Werke“ s. Haas 1983. Als Beispiel für eine Sammlung von Texten aus der Dialektliteratur, die sich (auch) 
tionell-mündlichen und mit elizierten Daten, zu denen deutlich mehr regionalsprachliche Analysen vorliegen. Neben den "traditionellen“ konzeptionell mündlichen Daten (Briefe, Tagebücher u. ä.) ${ }^{13}$ erfreuen sich die Daten der Neuen Medien (Internet, Handy) einer wachsenden Beliebtheit in der Variationslinguistik. ${ }^{14}$ Für regionalsprachliche Analysen werden dabei besonders häufig Chatdaten herangezogen. Bezogen auf das Medium handelt es sich bei diesen eindeutig um schriftliches Material, wohingegen die Konzeption eher auf Mündlichkeit verweist. ${ }^{15}$ Ein Vergleich von regionalsprachlichen Analysen zur Chatkommunikation in der Schweiz und in Deutschland deckt auf, dass die Aussagekraft des Materials deutlich von der soziolinguistischen Makrostruktur der Sprachgemeinschaft abhängt. Während etwa nach Siebenhaar (2005a) Dialekt in regionalen Chaträumen der Schweiz die unmarkierte Varietät darstellt, ergibt sich in Deutschland ein etwas anderes Bild: ${ }^{16} \mathrm{Hier}$ ist es nur bedingt möglich, (potentiell) regionalsprachliche Merkmale bzw. ihre Sprecher einer Region eindeutig zuzuordnen. Die Aussagekraft regionalsprachlicher Chatdaten aus dem deutschen Raum beschränkt sich i.d. R. auf die areal-soziale Verbreitung zumindest großregionaler Phänomene, die Bewertung und Salienz regionalsprachlicher Varianten, auf die sozialsymbolischen Funktionen von Dialektalismen und Regionalismen u. a.

Die nach wie vor größte Menge medial schriftlicher Daten, die für regionalsprachliche Analysen herangezogen werden, sind elizitierte Daten. Die Erhebung von medial schriftlichen Sprachdaten geht auf eine lange Tradition zurück, die ihren Anfang in der Dialektologie nimmt. Trotz wachsenden Methodenbewussteins und - damit einhergehend - häufig diskutierter Probleme,

als Untersuchungsgegenstand für regionalsprachliche Analysen anbietet, sei hier Cornelissen/Schmitt (Hg.) 1997 genannt.

13 S. etwa die Studie von Ziegler 2006, die auf „Zettelnachrichten“" aus bayerischen Schulklassen basiert. Wie Zieglers Analysen zeigen, gehören „,bestimmte Höflichkeitsformen und Akronyme ebenso wie der kontextspezifisch motivierte Gebrauch von Nonstandard- und Standardformen“ zum sprachlichen Repertoire der Schüler. „Der Dialekt stellt - wie zu erwarten - eine wesentliche kulturelle Ressource für die Konstruktion von Nähe dar. Allerdings gilt dies nicht für alle Dialektvarianten gleichermaßen, denn die einzelnen Dialektvarianten haben einen unterschiedlichen Status im Ausdrucksrepertoire der Jugendlichen. Insbesondere deuten die Ergebnisse darauf hin, dass Regionalformen, die großräumig verteilt und sowohl für den mittel- wie oberdeutschen Sprachraum typisch sind, stärker favorisiert werden als Dialektvarianten, die sich durch eine gewisse bairische Exklusivität auszeichnen.“

14 Zur Frage „Ist das World Wide Web ein Korpus?“ s. Lemnitzer/Zinsmeister (2006, S. $43 \mathrm{f}$.).

15 Zur Internetkommunikation im Allgemeinen (Nutzungsmotivation u. a.) s. Siever [u. a.] (Hg.) 2005, Beißwenger (Hg.) 2001. Zu sprachlichen Merkmalen von Chatdaten s. Storrer 2000, Androutsopoulos/Ziegler 2003, Ziegler 2005, Dürscheid 1999 und 2005. Zu linguistischen Aspekten der Mail- und SMS-Kommunikation s. Günther/Wyss 1996, Dürscheid 2002 und Androutsopoulos/Schmidt 2002.

16 Zur Schweiz s. Aschwanden 2001, Siebenhaar 2003 und 2005b. Zum deutschen Sprachraum s. etwa Christen/Tophinke/Ziegler 2005, Androutsopoulos/Ziegler 2003, Ziegler 2005 und Vogel 2003. 
die mit (insbesondere indirekt erhobenen) schriftlichen Befragungsdaten verbunden sind, ist das Verfahren nach wie vor sowohl in dialektologischen als auch regiolektal (,umgangssprachlich") orientierten Projekten sehr beliebt. ${ }^{17}$ Aktuelle regionalsprachliche Projekte, in denen schriftliche Befragungsdaten gesammelt werden, sind etwa der Syntaxatlas der Schweiz (s. Bucheli/Glaser 2002) und der „Atlas der deutschen Alltagssprache (ADA)“ (s. ElspaB 2005b). Letzterer dient auch als ein Beispiel für ein regionalsprachliches Forschungsprojekt, das die „Alltagssprache“ im deutschsprachigen Raum mittels Internetbefragungen in den Blick nimmt.

Unter den medial mündlichen Daten stellen sogenannte „Dokumentationsdaten" einen besonders wertvollen Typ dar. Ihnen ist eigen, dass der Zweck ihrer Aufnahme kein linguistischer ist, sondern diese Daten aus bestimmten nicht-linguistischen Gründen dokumentiert werden. Die Auswirkungen des Beobachterparadoxons werden somit minimiert bzw. ausgeschaltet. Als ein Beispiel für regionalsprachliche Daten dieses Typs seien hier Gesprächsdaten von Polizisten aus Notrufannahmen genannt. Diese werden aktuell im Rahmen eines Kooperationsprojekts des Forschungsinstituts für deutsche Sprache - Deutscher Sprachatlas (DSA) in Marburg und des Instituts für Deutsche Sprache (IDS) in Mannheim für das Bundeskriminalamt in Deutschland gesammelt und ausgewertet. Eine erste Auswertung liegt mit Kehrein (2006) vor.

Hinsichtlich der Elizitierung von medial mündlichem Sprachmaterial bedient sich die Regionalsprachenforschung besonders häufig der mündlichen Befragung, die unterschiedliche Grade der Standardisierung und Strukturiertheit annehmen kann. ${ }^{18}$ So weist etwa ein informelles und freies Gespräch zwischen dem Explorator und seinen Informanten i.d.R. einen geringeren Standardisierungsgrad als ein gesteuertes Einzel- oder Gruppeninterview auf. Noch standardisierter und strukturierter sind mündlich zu bewältigende Übersetzungsaufgaben oder Lückentexte, wie sie im Rahmen von Sprachatlasprojekten oder von Kompetenzerhebungen eingesetzt werden. ${ }^{19}$ Auch was die Datenerhebung per Beobachtung angeht, finden verschiedene Varianten der Methode Anwendung. Diese differieren in der Stellung des Beobachters zum Geschehen, im Standardisierungsgrad der Beobachtung, in der Sichtbarkeit des Beobachters oder in der Art der Beobachtungssituation (s. Lüdtke 2005). Die Grenze zwischen Beobachtungen bzw. Befragungen mit starkem Standardisierungsgrad und Experimenten ist fließend ${ }^{20}$, wobei letztere -

17 Anstelle einer ausführlichen Diskussion über die Vor- und Nachteile der indirekten Erhebung sei hier lediglich auf weiterführende Literatur verwiesen: Atteslander 2005, Glaser 2000, Bucheli/Glaser 2002, Lenz 2004, Cornips/Poletto 2005, Elspaß 2005 b.

18 Zur Methode der mündlichen Befragung s. Briggs 2005, Auer (1995, S. 431-433) und Thun 2005.

19 S. Bucheli/Glaser 2002, Cornips/Poletto 2005, Lenz (2003, S. 54-65) und Lenz 2004.

20 Dabei ist nach Auwärter (2005, S. 1077) als definierendes Kriterium für ein Experiment „nicht die besonders sorgfältige, kontrollierte Datenerhebung, und erst recht nicht die 
wie auch Testverfahren ${ }^{21}$ relativ selten als Erhebungsmethode eingesetzt werden.

In der Soziolinguistik [wie auch in der Regionalsprachenforschung; A. N. L.] stellen Experimente schon wegen der genannten externen Validitätsprobleme keine uneingeschränkt empfehlbare Forschungsmethode dar. Aber bei Berücksichtigung der Tatsache, dass die alternativen Methoden: Interviews, Tests und Beobachtung, und erst recht die pure Intuition, zum Teil die gleichen Mängel aufweisen, vielleicht zwar, besonders im Falle der Beobachtungsverfahren, höhere ökologische Validität aufweisen, dafür aber mangelnde intersubjektive Überprüfbarkeit und geringere Generalisierbarkeit, gehören Experimente auch nicht zu den diskriminierten Forschungsmethoden.

(Auwärter 2005, S. 1083)

Neben Dokumentations- und elizitierten oralen Sprachdaten sind in Abbildung (1) auch Sprachdaten aus „Funk und Fern(sehen)“ aufgeführt, die hinsichtlich der genannten Aspekte der Natürlichkeit und Authentizität einen speziellen Datentyp darstellen. Da auf diesen Typus nicht weiter eingegangen werden kann, sei hier exemplarisch auf regionalsprachliche Analysen verwiesen, die auf Radio- bzw. Fernsehsprachdaten basieren: Werlen 2000, Christen 2002 und Burger ( ${ }^{3} 2005$, S. 362-388).

\section{Regionalsprachliche Korpora des Deutschen (in Auswahl)}

Nach diesem Überblick über verschiedene Datenklassen, die für regionalsprachliche Fragestellungen herangezogen werden können, wird der Fokus auf regionalsprachliche Korpora gerichtet, die auf den vorgestellten Datenklassen aufbauen. Unter einem regionalsprachlichen Korpus wird hier eine abgeschlossene Menge an medial mündlichen oder medial schriftlichen Sprachdaten verstanden, die für die modernen Regionalsprachen des Deutschen bzw. für regionalsprachliche Spektrumsausschnitte (Varietäten) repräsentativ sind, durch Metadaten erschlossen sind und (im weiteren Sinne) computertechnisch aufbereitet vorliegen. ${ }^{22}$

Im Rahmen dieses Beitrags können nur einige ausgewählte regionalsprachliche Korpora ausführlicher diskutiert werden. Da sich die Mehrheit regionalsprachlicher Analysen - trotz wachsenden Interesses an insbesondere Internetkommunikationsdaten - nach wie vor auf elizierte Daten stützt, wird sich die folgende Diskussion auf regionalsprachliche Korpora dieses Datentyps konzentrieren. ${ }^{23} \mathrm{Zu}$ einer ersten Einordnung dient Abbildung (2), aus der die Datenbasis der im Zentrum stehenden Korpora ablesbar ist.

Laborsituation [anzusehen; A. N. L.], sondern ein Design, das wesentlich die ,Manipulation', die systematische Variation von Faktoren vorsieht - oder im Grenzfall die Analyse der systematischen Variation dieser Faktoren durch ,natürliche Vorkommnisse'." $\mathrm{Zu}$ variationslinguistischen Tests s. Spolsky 2005.

22 Zu einem ähnlichen Korpusbegriff s. McEnery/Wilson (1996, S. 21). Zur Definition und Abgrenzung linguistischer Korpora s. auch Lemnitzer/Zinsmeister (2006, S. 40-44).

${ }^{23}$ Hinweise zu weiteren, hier unberücksichtigten Sprachkorpora des Deutschen liefern 
(2) Regionalsprachliche Korpora des Deutschen (in Auswahl) und ihre Datenbasen

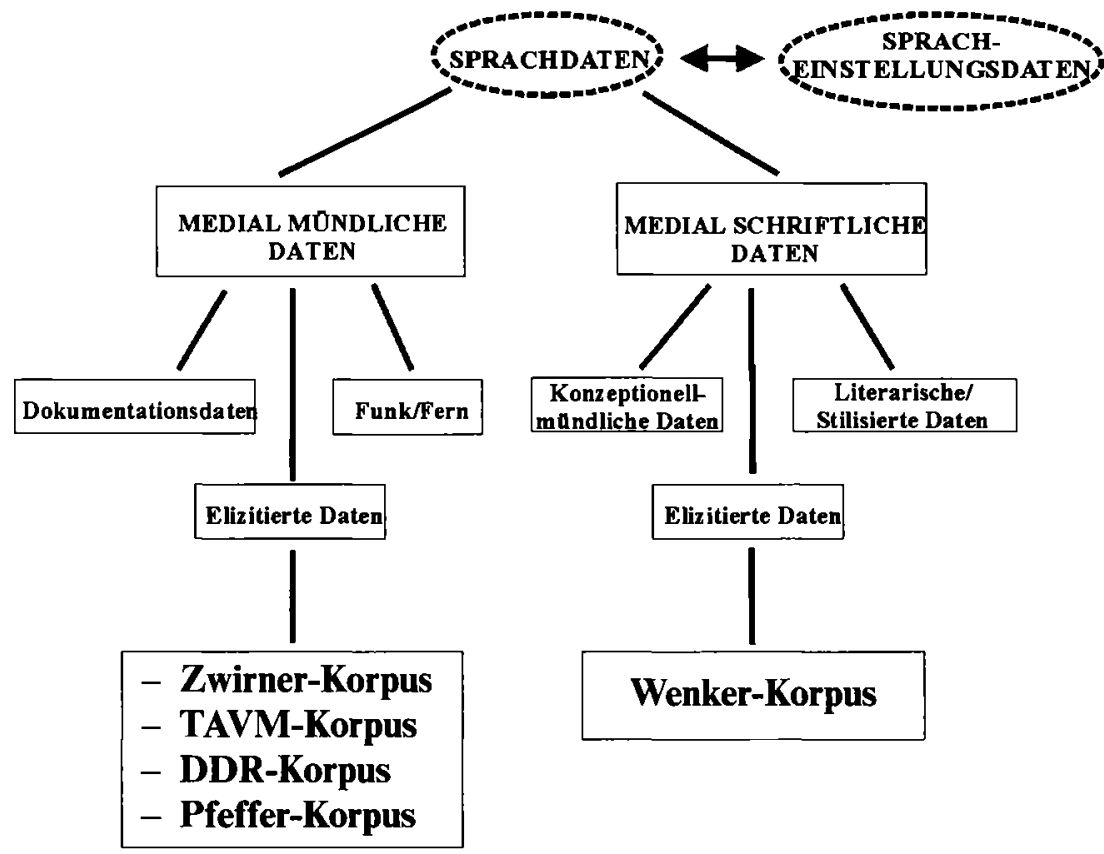

Abb. 2

Bei den in Abbildung (2) aufgeführten Korpora medial mündlicher Sprachdaten handelt es sich um Tonkorpora und zugehörige Transkripte, die in die „Datenbank Gesprochenes Deutsch (DGD)“ des Instituts für deutsche Sprache in Mannheim eingebunden sind bzw. noch werden und für wissenschaftliche Zwecke frei zugänglich sind. ${ }^{24}$

Die Datenbank Gesprochenes Deutsch (DGD) ist die digitale Realisierungsform des Deutschen Spracharchivs (DSAv). Ihre Aufgabe ist, im Laufe der Zeit den Gesamtbestand des DSAv und weitere einzuwerbende Korpora digital zu archivieren und diese Materialien des Archivs in elektronischer Form für interne wie für externe Nutzer zu erschließen und variabel zugänglich zu machen. Die DGD erfüllt damit einerseits Archivfunktion, auf der anderen Seite ist sie ein Forschungsinstrument.

(Fiehler/Wagener 2005, S. 139)

Schwitalla/Wegstein 2005, Storrer 2005, Schlobinski (1996, S. 72-74), Wagener/Bausch 1997, Glas/Ehlich 2000 und Lemnitzer/Zinsmeister 2006. S. auch die Literaturangaben in Fn. 25.

24 Im Gegensatz dazu steht etwa das oben erwähnte Polizisten-Korpus aus Notrufannahmen, das aktuell in Mannheim und Marburg aufgebaut wird, aus datenschutzrechtlichen Gründen nicht öffentlich zur Verfügung. 
Ausführlichere Informationen über die vier DGD-Korpora sind in Tabelle (3) zusammengestellt. Die Angaben sind v. a. den Webseiten der „Korpusstelle Gesprochenes Deutsch“ des Instituts für Deutsche Sprache in Mannheim entnommen (http://www.dsav-oeff.ids-mannheim.de [Stand: April 2006]).

(3) Regionalsprachliche Tonkorpora der „Datenbank Gesprochenes Deutsch (DGD)“ (in Auswahl) (http://dsav-oeff.ids-mannheim.de) ${ }^{25}$

\begin{tabular}{|c|c|c|c|c|}
\hline & $\begin{array}{l}\text { „Zwirner- } \\
\text { Korpus“ }\end{array}$ & $\begin{array}{l}\text { „TAVM- } \\
\text { Korpus }^{626}\end{array}$ & $\begin{array}{l}\text { „DDR- } \\
\text { Korpus“ }\end{array}$ & $\begin{array}{l}\text { "Pfeffer- } \\
\text { Korpus }\end{array}$ \\
\hline $\begin{array}{l}\text { Intendierte } \\
\text { Varietät/ } \\
\text { Sprechlage }\end{array}$ & $\begin{array}{l}\text { Dialekt } \\
(+, \text {,Umgangs- } \\
\text { sprache“) }\end{array}$ & Dialekt & $\begin{array}{l}\text { Dialekt } \\
(+, \text {,Umgangs- } \\
\text { sprache“ })\end{array}$ & $\begin{array}{l}\text { "städt. } \\
\text { Umgangs- } \\
\text { sprache“ }\end{array}$ \\
\hline $\begin{array}{l}\text { Aufnahme- } \\
\text { region }\end{array}$ & $\begin{array}{l}\text { v. a. alte } \\
\text { Bundesrepublik }\end{array}$ & $\begin{array}{l}\text { ehemalige } \\
\text { deutsche Ost- } \\
\text { gebiete }\end{array}$ & DDR & $\begin{array}{l}\text { deutsch- } \\
\text { sprachiger } \\
\text { Raum } \\
\end{array}$ \\
\hline Ortsnetz & $\begin{array}{l}\text { ca. } 1000 \text { Orts- } \\
\text { punkte }\end{array}$ & $\begin{array}{l}\text { ca. } 900 \text { Orts- } \\
\text { punkte }\end{array}$ & $\begin{array}{l}440 \text { Orts- } \\
\text { punkte }\end{array}$ & 57 Städte \\
\hline $\begin{array}{l}\text { Anzahl der } \\
\text { Aufnahmen }\end{array}$ & 5857 & 984 & 1625 & 398 \\
\hline $\begin{array}{l}\text { Aufnahme- } \\
\text { datum }\end{array}$ & v. a. $1955-1960$ & $1962-1965$ & $1960-1964$ & 1961 \\
\hline $\begin{array}{l}\text { Aufnahme- } \\
\text { dauer }\end{array}$ & $\begin{array}{l}\text { jew. ca. } 12 \text { min } \\
\text { (insges. } \\
\text { ca. } 1092 \mathrm{~h} \text { ) }\end{array}$ & $\begin{array}{l}\text { jew. ca. } 28 \mathrm{~min} \\
\text { (insges. } \\
\text { ca. } 462 \mathrm{~h} \text { ) }\end{array}$ & $\begin{array}{l}\text { insges. } \\
\text { ca. } 393 \mathrm{~h}\end{array}$ & $\begin{array}{l}\text { jew. ca. } 12 \mathrm{~min} \\
\text { (insges. } \\
\text { ca. } 79 \mathrm{~h} \text { ) }\end{array}$ \\
\hline Inhalt & $\begin{array}{l}\text { v. a. Dialoge, } \\
\text { Erzählungen }\end{array}$ & $\begin{array}{l}\text { Erzählungen, } \\
\text { Wenkersätze, } \\
\text { Wochentage }\end{array}$ & $\begin{array}{l}\text { Erzählungen, } \\
\text { Dialoge, } \\
\text { Vergleichstexte }\end{array}$ & $\begin{array}{l}\text { Dialoge, } \\
\text { Erzählungen }\end{array}$ \\
\hline Sprecher & $\begin{array}{l}\text { je } 3 \text { autochthone } \\
\text { Dialektsprecher } \\
\text { ( } 3 \text { Generationen) } \\
+3 \text { Aussiedler }\end{array}$ & $\begin{array}{l}\text { (heimat)dialekt- } \\
\text { kompetente } \\
\text { Vertriebene } \\
\text { (>60 Jahre) }\end{array}$ & $\begin{array}{l}\text { je } 3 \text { autochthone } \\
\text { Dialektsprecher } \\
\text { ( } 3 \text { Generationen) }\end{array}$ & $\begin{array}{l}403 \text { „Umgangs- } \\
\text { sprache- } \\
\text { sprecher“ } \\
(185 w+218 \mathrm{~m})\end{array}$ \\
\hline $\begin{array}{l}\text { Ton/Transkr. } \\
\text { (synchron.) }\end{array}$ & 2032 & 261 & - & 398 \\
\hline
\end{tabular}

${ }_{25} \mathrm{Zu}$ den in (3) aufgeführten sowie zu weiteren Korpora der DGD s. Wagener/Bausch 1997 und Wagener 2002. Ausführliche Informationen zum Aufbau der „Datenbank Gesprochenes Deutsch (DGD)" geben u. a. Fiehler/Wagener 2005 und Wagener 2005.

26 In der DGD ist das TAVM-Korpus (,Tonaufnahme der Vertriebenenmundarten“) unter der Bezeichnung "Deutsche Mundarten: ehemalige deutsche Ostgebiete" und mit der Korpussigle „OS“ aufgeführt. 
Die Sprachdaten der vier DGD-Korpora wurden überwiegend zwischen 1955 und 1965 erhoben. Das weitaus größte Korpus der DGD stellt mit fast 6000 Aufnahmen an ca. 1000 ländlichen Ortspunkten im Gebiet der alten Bundesrepublik das „Zwirner-Korpus“ dar. ${ }^{27}$ Indem Eberhard Zwirner ,als Mundartsprecher je eine alte Person aus der ländlichen Bevölkerung[,] dann je eine Person mittleren Alters aus kleinbürgerlichen Kreisen und schließlich je ein[en] Sprecher aus der schulentlassenen Jugend" berücksichtigte, erreichte er, dass „nicht nur die Mundarten, sondern auch die ländliche und kleinstädtische Umgangssprache und gleichzeitig verschiedene Stadien der bereits im Gang befindlichen und weitgehend vom Alter abhängigen sprachlichen Ausgleichsvorgänge erfaßt" wurden (Zwirner 1956, S. 12). Falls an einem Aufnahmeort auch Aussiedler aus den ehemaligen ostdeutschen Gebieten anzutreffen waren, nahm Zwirner auch von diesen drei Sprecher auf, und zwar aus der lokal am „häufigsten vertretene[n] landsmannschaftliche[n] Gruppe“ (Bethge/Zwirner 1958, S. 16). Die von Zwirner eingesetzte Erhebungsmethode war überwiegend die der (möglichst) wenig strukturierten Befragung. Die Aufnahmen umfassen vor allem Dialoge und initiierte Erzählmonologe. Da die Aussiedler aus den ehemaligen deutschen Ostgebieten im Rahmen des Zwirner-Projektes nur unsystematisch berücksichtigt werden konnten, diente die „Tonaufnahme der Vertriebenenmundarten (TAVM)“ der systematischen Füllung der arealen Lücken, wobei der Fokus des TAVM-Projektes auf der Erhebung der Basisdialekte lag (s. Bellmann 1964 und 1970). Neben den Dialekten im Osten des deutschen Sprachgebiets fanden auch deutsche Sprachinseln in Ost- und Südosteuropa Berücksichtigung.

$\mathrm{Zu}$ den bereits digitalisierten Korpora des Deutschen Spracharchivs in Mannheim gehört auch das sogenannte „DDR-Korpus“ ${ }^{28}$, das in seiner methodischen Anlage eng an das Zwirner-Korpus angelehnt ist, insofern als bei der Datenerhebung drei einheimische Sprecher aus verschiedenen Altersgruppen pro Aufnahmeort in der DDR berücksichtigt wurden. Im DDR-Projekt, das die Zwirner-Aufnahmen in den neuen Bundesländern komplementiert, wurden in mehreren Regionen auch bzw. seltener (beispielsweise in der Berliner Region) nur ,umgangssprachliche“ Aufnahmen erstellt. Gemeinsam decken das Zwirner-, das TAVM- und das DDR-Korpus die deutschsprachigen Dialekte in der Mitte des letzten Jahrhunderts in einem relativ dichten Ortsnetz ab.

Ergänzend zu diesen drei Korpora sei hier das „Pfeffer-Korpus“ genannt, das zur Dokumentation "städtischer Umgangssprachen“ erstellt wurde (s. Pfeffer/Lohnes 1984). An insgesamt 57 Städten im deutschsprachigen Raum wurden im Jahr 1961403 Sprecher aufgenommen, deren Sprechweise

27 Ausführlichere Informationen zur Konzeption von Zwirners Großprojekt geben Zwirner 1956 und 1958 sowie Zwirner/Bethge 1958 und Wagener/Bausch (1997, S. 112-114).

28 Zum zugrunde liegenden Projekt „Tonbandaufnahme der deutschen Mundarten in der Deutschen Demokratischen Republik“s. Schädlich/Große 1961. 
als „umgangssprachlich“ eingestuft wurde. Im Vergleich zu den Zwirner-, TAVM- und DDR-Aufnahmen, die mehrheitlich im dialektalen bis mittleren Bereich des variativen regionalsprachlichen Gesamtspektrums angeordnet werden können, ist das Pfeffer-Korpus - obwohl auch hier die „Varietätenbreite [...] relativ groß ist“" (Spiekermann 2005, S. 107) - insgesamt als standardnäher zu bewerten.

Der Bearbeitungsstand der einzelnen DGD-Korpora - d.h. ihrer dokumentarischen Daten ${ }^{29}$, Tonaufnahmen und Transkriptionen - ist sehr unterschiedlich. Wie die „Bestandsübersicht“ der DGD aktuell (April 2006) angibt, liegen die Tonaufnahmen und Transkripte des Pfeffer-Korpus komplett digitalisiert und miteinander synchronisiert vor (http://dsav-wiss.ids-mannheim. de/DSAv/KORPORAB.HTM). Für das Zwirner-Korpus sind bereits etwa knapp die Hälfte aller Transkripte mit aligniertem Ton verfügbar, für das TAVM-Korpus ca. ein Drittel. Die Alignierung der Transkripte ermöglicht die gezielte Tonwiedergabe zum gewählten Transkriptausschnitt. Bei den verfügbaren Transkriptionen handelt es sich i.d. R. um literarische, weitgehend an der standardsprachlichen Orthographie orientierte Transkripte, die als reine Textdateien vorliegen, in denen mit den Suchbefehlen des Volltextretrievalinstruments COSMAS II recherchiert werden kann (vgl. Fiehler/Wagener 2005, S. 141). Die DDR-Aufnahmen und -transkripte sind bislang noch nicht online zugänglich. Alle vier Korpora sind durch ausführliche Metadaten erschlossen („Dokumentationen“), die u. a. soziodemographische Informationen zu den Sprechern, zu Inhalt und Länge der Tonaufnahmen, zur vertikalen Positionierung der realisierten Sprechlagen auf der Dialekt-StandardAchse und zur dialektgeographischen Zuordnung der Aufnahmen enthalten.

Da der Fokus dieses Beitrags auf der variationslinguistischen Analyse regionalsprachlicher Korpora liegt, kann der Blick auf medial mündliche Sprachkorpora des Deutschen nur ausschnitthaft sein. Weitere, hier nicht genannte Sprachkorpora - bestehend aus Tonaufnahmen und/oder phonetischen Transkriptionen - sind bzw. werden u. a. in den folgenden Instituten und Archiven gesammelt, archiviert und aufbereitet.

(4) Institute/Archive medial mündlicher Sprachkorpora des Deutschen (ohne Anspruch auf Vollständigkeit)

- Amt für rheinische Landeskunde (ARL) des Landschaftsverbandes Rheinland (LVR) (Bonn)

- Archiv für Gesprochenes Deutsch (AGD) (IDS Mannheim)

- Bayerisches Archiv für Sprachsignale (BAS) der Ludwig-MaximiliansUniversität München

29 „Eine Recherche in den dokumentarischen Daten gibt einen Überblick über die Bestände oder kann je nach der vom Nutzer formulierten Anforderung bestimmte Gruppen von Sprechern, bestimmte Gesprächstypen, Sprache aus verschiedenen Sprachlandschaften u. ä. suchen." (Fiehler/Wagener 2005, S. 140). 
- Berliner Lautarchiv der Humboldt-Universität Berlin

- Deutsches Rundfunkarchiv (DRA) (Wiesbaden/Babelsberg)

- Hoerburger-Archiv an der Universität Regensburg

- Institut für Phonetik und digitale Sprachverarbeitung der ChristianAlbrechts-Universität Kiel

- Max Kade Institute for German American Studies (Madison)

- Phonogrammarchiv der Österreichischen Akademie der Wissenschaften (Wien)

- Phonogrammarchiv der Universität Zürich

- Schallarchiv der Philipps-Universität Marburg

- „Ulmer Textbank“ der Universität Ulm

- Unterfränkisches Dialektinstitut (UDI) der Julius-Maximilians-Universität Würzburg ${ }^{30}$

- Wossidlo-Archiv (Institut für Volkskunde) der Universität Rostock

Das größte medial schriftliche regionalsprachliche Korpus des Deutschen ist das „Wenker-Korpus“. Es besteht aus ca. 50.000 zweiseitigen Fragebogen, die v. a. Ende des 19. Jahrhunderts von dem Marburger Dialektologen Georg Wenker (1852-1911) erhoben wurden. Wenker bediente sich des Verfahrens der indirekten Befragung, was angesichts der erhobenen Datenmasse sicher die einzig mögliche Erhebungsform darstellte. Im Rahmen seines sukzessive ausgeweiteten Atlasprojektes schickte er - beginnend im Jahr 1876 - an alle Schulorte des ehemaligen deutschen Reichs einen Fragebogen bestehend aus 38 bis 42 Sätzen mit der Bitte an die dortigen Volksschullehrer, ,eine Uebersetzung der einliegenden hochdeutschen Sätzchen in die ortsübliche Mundart" auf den jeweiligen Wenkerbogen einzutragen, wobei die Lehrer aufgefordert waren, die Übersetzung „durch einen oder einige geeignete Schüler anfertigen" $\mathrm{zu}$ lassen. ${ }^{31}$

Die Wenkerbogen stellen die Grundlage von Wenkers „Sprachatlas des deutschen Reichs“ (1888-1923) dar. Auf den 576 zusammengesetzten Gesamtkarten (1643 Teilkarten) des Atlasses sind jedoch nur ausgewählte Lemmata der Wenkerbogen kartiert. Auch sind weiterführende Informationen (Geburtsort des Lehrers, Angaben zu den für das Ausfüllen des Bogens ver-

30 Am UDI entsteht aktuell die „Bayerische Dialektdatenbank (Baydat)“, die auf den Transkriptionen des Bayerischen Sprachatlasses (BSA) basiert, dessen Daten $1984 \mathrm{ff}$. erhoben wurden. „Im Rahmen von Baydat soll das kostbare BSA-Material [...] zukunftssicher archiviert, nach modernen Kriterien aufbereitet, für EDV- und Online-Recherchen zur Verfügung gestellt werden und auf diese Weise allen Interessierten einen strukturierten Zugriff bieten." (http://www.uni-wuerzburg.de/germanistik/spr/suf/ baydat-udi/baydat.html) [Stand: 07.06.2006]

31 Zitiert aus Wenkers Anschreiben an die Lehrer von 1879/1880 (Erhebungsbogen für Nord-, Mittel- und Süddeutschland). Mit Herrgen/Schmidt (in Bearb.) ist zu betonen: „Im Rahmen sprachdynamischer Analysen kann es [...] notwendig sein, zu kontrollieren, für welche Sprechergruppe die lokalen Sprachdaten überhaupt Geltung beanspruchen können." 
antwortlichen Personen u. a.) allein auf den Rückseiten der Bogen festgehalten. Trotz der Schwierigkeiten, die etwa die Kurrentschrift bei der Analyse der Daten verursacht, sind die Wenkerbogen als eine zentrale, die Wenkerkarten ergänzende Datenquelle für die Dialekte Ende des 19. Jahrhunderts anzusehen. Im von der DFG geförderten Projekt „Digitaler Wenker-Atlas (DiWA)“ sind deshalb nicht allein die Wenkerkarten erstmals publiziert worden, und zwar online, sondern auch die Wenkerbogen wurden digitalisiert und werden aktuell - in Form von Bilddateien - sukzessive online gestellt (http://www. diwa.info). ${ }^{32}$

„Wenker-Korpus“ (http://www.diwa.info)

Intendierte Varietät/Sprechlage

Erhebungsregion

Ortsnetz

Erhebungszeitraum

Umfang

Inhalt

Informanten

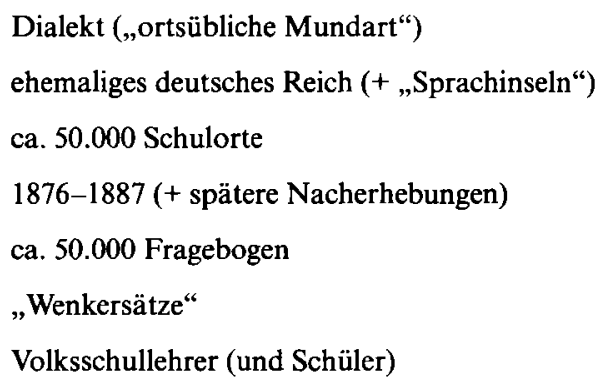

Die Datenbankstruktur des DiWA stellt verschiedene Abfrage- und Registerfunktionen für alle bereits bzw. zukünftig integrierten Daten zur Verfügung. $\mathrm{Zu}$ diesen gehören neben den ca. 100.000 digitalisierten Fragebogenseiten (jeweils Vorder- und Rückseite) die Wenkerkarten, die Erhebungsorte und ihre (alten bzw. aktuellen) Namen, Karten moderner Regionalatlanten, weitere (historische, soziodemographische u. a.) Karten-,,Interpretamente“, Tonaufnahmen, bibliographische und sonstige ergänzende Informationen. Die Wenkerbogen sind in gleicher Weise einsehbar wie die georeferenzierten Wenkerkarten. Beim Anklicken eines Ortspunktes erscheint der jeweilige Wenkerbogen in einem neuen Fenster, in dem beliebige Zoomausschnitte des Bogens definiert werden können.

\section{Regionalsprachliche Korpora des Deutschen in der Analysepraxis}

Im Folgenden werden drei der vorgestellten Korpora - zum einen das Zwirner- und das Pfeffer-Korpus, zum anderen das Wenker-Korpus - zur Analyse von zwei regionalsprachlichen Phänomenen aus dem lautlichen bzw. grammatischen Bereich herangezogen. Wie die beiden Beispielanalysen zeigen

32 Zur Konzeption und Technik des DiWA s. Herrgen/Lenz 2003, Herrgen/Lenz/Pennay 2003, Kehrein/Lameli/Nickel 2005. 
werden, bedingt die deutlich differierende Anlage der Korpustypen - zum einen medial mündliche, zum anderen medial schriftliche Sprachdaten - und auch die deutlich differierende Zugänglichkeit - einmal Transkriptionen in Form von Textdateien, zum anderen Bilddateien mit reichem Zusatzmaterial unterschiedlichste Analysemöglichkeiten und -methoden.

\section{Beispiel 1: Variationslinguistische Analyse auf der Grundlage des Wenker-Korpus}

Der Fokus der ersten Analyse wird auf spezifischen Problemen des Wenkermaterials liegen, die insbesondere mit der laiensprachlichen Verschriftung der Wenkersätze in Zusammenhang stehen. Wie bereits Wrede (1895) betont

„[darf man] die Übersetzungen und demgemäß das, was auf den fertigen Karten steht, [...] nicht als phonetisch genaue Dialektwiedergaben auffassen, sondern jede Karte erfordert, grade so wie jede alte Handschrift, eine besondre, häufig recht schwierige Interpretation, wie die darauf verzeichneten Sprachformen phonetisch zu übertragen sind.“

(Wrede 1895, S. 37)

Das zu diskutierende Phänomen betrifft die Reflexe des westgermanischen $g$ im Moselfränkischen. In diesem Dialektraum stehen den standardsprachlichen plosivischen Realisierungen [g] bzw. [k] in- und auslautend regionalsprachliche Spiransvarianten gegenüber. ${ }^{33}$ Beispielsweise trifft der standardsprachliche Plosiv [k] $(<\mathrm{g}>$ im Silbenauslaut und präkonsonantisch) im Moselfränkischen auf die Spiranten [ $]$ ] bzw. [x], wie sie auslautend etwa in

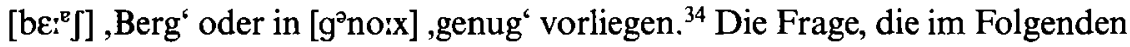
diskutiert werden soll, ist die nach der Dynamik der moselfränkischen Spiranten, das heißt nach Stabilität, Progression oder Abbau im Laufe der letzten 100 Jahre. Um diese Frage (zumindest ansatzweise) zu beantworten, werden die Wenkerdaten (Ende des 19. Jahrhunderts) mit anderen regionalsprachlichen Daten aus den vergangenen 120 Jahren kontrastiert.

Da die Graphien der Wenkerlehrer unverändert von den Fragebogen auf die Wenkerkarten übertragen worden sind, kommt ein Blick in die Wenkerkarten einem Blick in die Wenkerbogen (annähernd) ${ }^{35}$ gleich, denn

33 Zur standardsprachlichen Aussprachenorm von $<\mathrm{g}>$ s. Duden $\left({ }^{4} 2000\right.$, S. 84-86). Zu den dialektalen Verhältnissen s. Schirmunski (1962, S. 309-316).

34 Neben der alveolaren Spirans [ $\left.\int\right]$ findet sich in den rezenten westmitteldeutschen Regionalsprachen $u$. a. auch koronalisiertes [c]. S. ausführlicher Herrgen 1986 und Lenz (2003, S. $148-158$ u. 165-177).

35 Mit Kehrein/Lameli/Nickel (2005) ist zu betonen: „Das Ansetzen von Isoglossen ist ein subjektives Verfahren, das auf der wissenschaftlichen Analyse des Kartenzeichners basiert und seinem linguistischen Urteilsvermögen unterliegt. Daher ist für linguistisch ungeschulte Rezipienten bei der Arbeit mit den im DiWA publizierten Wenker-Karten grundsätzlich mit Bedacht vorzugehen. Die kombinierte Flächen- und Punktsymbolkartierung evoziert durch die mit markanten Farben dargestellten Leitformen oft einen ersten plakativen Eindruck der Kartensituation, der aber gewissenhaft hinterfragt und 
[w]as die technische Herstellung der Sprachatlaskarten betrifft, so sei nur daran erinnert, dass die Kartenbilder aus dialektischen Übersetzungsformularen gewonnen sind, [...] und dass die in diesen [...] Formularen gebotenen Sprachformen ganz mechanisch nach der Originalschrift, ohne theoretisierende Besserungen oder dergl., in die Karten eingetragen wurden.

(Wrede 1895, S. 34)

In (6) ist ein Ausschnitt der Wenkerkarte ,,(ge)nug (Satz 16)“ aus dem linksrheinischen Moselfränkischen abgebildet (eingebettet in die DiWA-Benutzeroberfläche), wobei die Städte Trier und Koblenz zur Orientierung hervorgehoben sind. Kartiert ist die zweite Silbe von genug. Obwohl es sich um einen moselfränkischen Kartenausschnitt handelt, sind nördlicheres „nog“ und südlicheres ,nug“ (im moselfränkisch-rheinfränkischen Übergangsgebiet) als Leitformen für die durch - im Original farbige - Isoglossen abgegrenzten Areale kartiert. Innerhalb der beiden Areale weisen verschiedene Zusatzsymbole auf lokale Abweichungen der Lehrergraphien von den Leitformen „nog“ und „nug" hin. In der - hier nicht abgebildeten - Legende wird ersichtlich, dass es sich bei senkrechten schwarzen Strichsymbolen um ch-Graphien der Lehrer handelt, die aber nur vereinzelt auftauchen. Dies widerspricht auf den ersten Blick der Erwartung, in den abgebildeten Regionen flächendeckend auf Spiranten zu treffen. Andere Wenkerkarten bzw. Wenkerbogen, die zur Analyse der dialektalen Reflexe von westgermanisch $g$ herangezogen werden können, bestätigen jedoch, dass die $g$-Allographen in den Transliterationen der moselfränkischen Lehrer keine Ausnahme darstellen bzw. die ch-Graphien jeweils deutlich seltener auftreten.

Zur Interpretation dieser Befunde sind weitere regionalsprachliche Quellen heranzuziehen. $\mathrm{Zu}$ diesen gehören nicht zuletzt „Ortsgrammatiken“ vom Ende des 19. Jahrhunderts bzw. Anfang des 20. Jahrhunderts, die als besonders wertvolle, das Wenkermaterial ergänzende Datenquellen anzusehen sind. Für die dialektalen Reflexe des alten $g$ im moselfränkischen Sehlem gibt z. B. Ludwig (1906) folgende Beschreibung:

[...] inlautend erscheint mhd. $g$ l. als stimmhafte Spirans vor stimmh. Laut a) nach Hartgaumenvokalen sowie nach $l, r$ palatal $=j,[\ldots]$; b) nach Flachzungen- und Weichgaumenvokalen velar $=3,[\ldots] ;$ 2. als stimmloser Reibelaut vor stimml. Konson., je nach der Natur des vorhergehenden Vokals $[\ldots]$, bald als palatales $\chi,[\ldots]$ bald als velares $x,[\ldots]$; c) auslautendes, altes germ. $g$ ( $>$ mhd. $c$ ), wie auch das nach Apokope eines Schluss-e an die Endung rückende $g$ ist nach obiger Regel (vgl. 2) bald $\chi,[\ldots]$ bald $x,[\ldots]$; d) intervokalisches $g$ ist bisweilen völliger Unterdrückung ausgesetzt [...].

(Ludwig 1906, S. 43 f.)

erst durch kleinräumige Betrachtung des Analysegebiets belegt werden muss. Es ist wichtig, die impliziten Interpretationsleistungen der Kartenzeichner nachzuvollziehen, die durch die Georeferenzierung des historischen Kartenmaterials offen gelegt werden können, [...].“ 
(6) Moselfränkischer Ausschnitt der Wenkerkarte ,(ge)nug (Satz 16)“ (eingebettet in die DiWA-Benutzeroberfläche) (http://www.diwa.info)

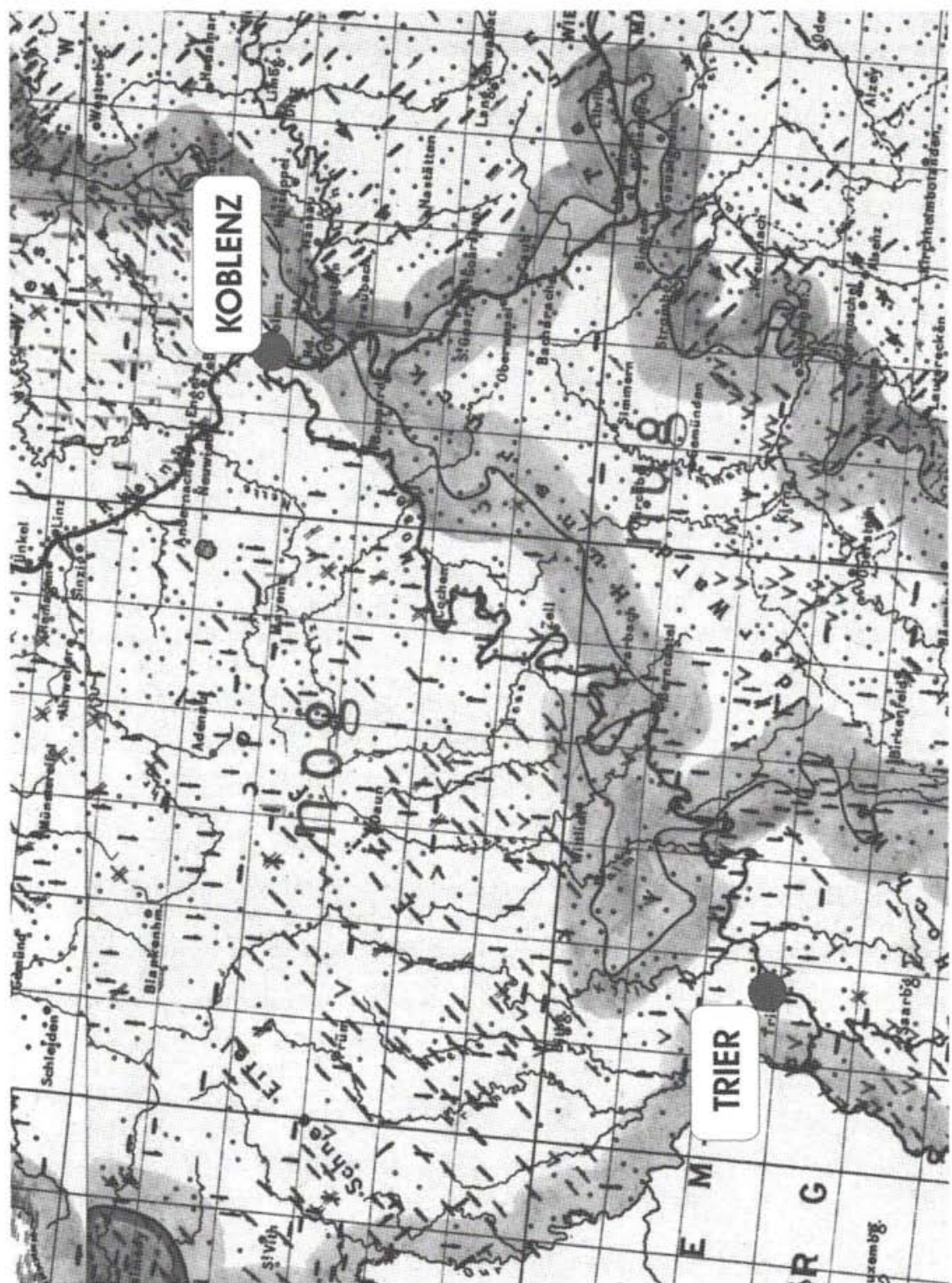

․

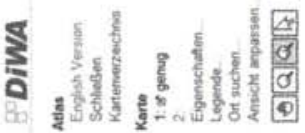


Neben den i.d.R. von phonetisch geschulten Dialektologen erstellten Ortsmonographien weisen auch regionalsprachliche Tonaufnahmen (spätestens) aus der zweiten Hälfte des 20. Jahrhunderts die Spiranten flächendeckend für das Moselfränkische nach. Mit den Zwirner-Aufnahmen der DGD sind hier Wenkersatzaufnahmen des Forschungsinstituts für deutsche Sprache - Deutscher Sprachatlas in Marburg zu nennen, die aktuell in DiWA integriert und dort ortspunktgenau über die Wenkerkarten abgerufen werden können (www. diwa.info). Ebenso belegen der „Mittelrheinische Sprachatlas (MRhSA)“, ein moderner Regionalatlas mit Dialektdaten aus den $1980 \mathrm{ern}^{36}$, sowie aktuelle variationslinguistische Untersuchungen eine stabile Verteilung der Spiranten in den Dialekten und auch in den Regiolekten („Umgangssprachen“) des Moselfränkischen in der zweiten Hälfte des 20. Jahrhunderts (s. Lenz 2003, S. 148-158). Die gesammelten Quellen stützen die Thesen, dass die Spiransvarianten erstens auch zur Wenkerzeit in den Dialekten des Moselfränkischen verankert waren und zweitens bis heute ein abbauresistentes Dialekt- und Regiolektmerkmal des Moselfränkischen darstellen, das bis in standardnahe Sprechlagen hinein Verwendung findet. ${ }^{37}$

Vor dem Hintergrund dieser Ergebnisse werden die g-Allographen der Wenkerlehrer im Moselfränkischen nicht als Belege für plosivische Lautvarianten zur Wenkerzeit interpretiert, sondern als Spiranten, die von den Lehrern mit dem Schriftzeichen $\langle\mathrm{g}\rangle$ wiedergegeben wurden. Es ist davon auszugehen, dass die Lehrer (und Schüler) der Wenkerzeit auch in ihrer Leseaussprache das Schriftzeichen $<\mathrm{g}>$ spirantisiert ausgesprochen haben. Diese Interpretation einer spirantischen $<\mathrm{g}>$-Aussprache im intendierten „Hochdeutsch“ westmitteldeutscher Sprecher ist nicht neu. ${ }^{38}$ Beispielsweise geht Schirmunski (1962) in seiner „Deutschen Mundartkunde“ auf das Phänomen ein:

36 Beim MRhSA handelt es sich um einen bidimensionalen Regionalatlas, insofern als neben einer alten immobilen Sprechergruppe auch jüngere Berufspendler bezüglich ihrer Dialektkompetenz befragt wurden. S. ausführlicher Bellmann 1994. Auch im intergenerationellen Vergleich weisen die dialektalen Spiranten eine (relative) Stabilität im Moselfränkischen bzw. sogar eine Ausweitung ins Rheinfränkische auf. S. hierzu etwa die MRhSA-Karten 391/1-2, 392/1-2 und 393/1-2.

37 Wie u. a. für die Region Wittlich (Eifel) nachgewiesen werden konnte, korreliert die Abbaustabilität der $g$-Spiranten mit ihrer Stellung und ihrem lautlichen Kontext. Die größte Stabilität weist die Spirans [x] im Silbenauslaut auf. Im Moselfränkischen ist sie in dieser Position zu den quintären und damit zu den abbauresistentesten Merkmalen der Regionalsprache zu rechnen. Vgl. Lenz (2003, S. 186-191).

38 S. neben Schirmunski (1962, S. 315 f.) auch besonders Mihm (2004, S. 190) und Mattheier (1979, S. 220). Ein Blick in die Forschungsliteratur belegt, dass auch in älteren Texten der Vorwenkerzeit (etwa im Diminutivsuffix -gen) häufig eine Allographie von $<\mathrm{g}>$ und $<\mathrm{ch}>$ als spirantische Repräsentanten anzunehmen ist. Vgl. Wegera 2000, Elspaß (2000, S. 254, Fnn. 13 und 14) sowie Elspaß (2005a, S. 340-345). An dieser Stelle danke ich noch einmal herzlich Stephan Elspaß (Augsburg) und Arend Mihm (Duisburg), die mich auf ergiebige Literatur zum Phänomen aufmerksam gemacht haben. 
Spirantische Aussprache des intervokalischen und des auslautenden $g$ ist sowohl in Nord-als auch besonders in Mitteldeutschland bis heute als lokale Ausspracheweise der Literatursprache erhalten geblieben und wird weitgehend auch weiterhin beibehalten. [...] Goethe reimte als gebürtiger Frankfurter (Main) entsprechend seiner Aussprache Buch : genug, [...] zeigen : reichen [...]. In Verbindung mit der Frage der Schaffung einer einheitlichen literatursprachlichen Aussprachenorm für das Deutsche entspann sich noch im letzten Viertel des 19. Jhs. in den philologischen und pädagogischen Zeitschriften eine äußerst heftige Polemik über die richtige Aussprache des $g$. Eine bedeutende Gruppe der Beteiligten, und zwar vorwiegend solche, die aus Mitteldeutschland stammten (darunter der bekannte Phonetiker W. Vietor) verteidigte die mitteldeutsche Norm (explosives $g$ - im Wortanlaut, Reibelaut im In- und Auslaut) als wünschenswerten Kompromiß zwischen den niederdeutschen und den oberdeutschen Aussprachegepflogenheiten. Doch die Autorität der ,Bühnenaussprache‘ erlangte nach langem Streit das oberdeutsche Verschluß-g; [...].

(Schirmunski 1962, S. 315f.)

Angesichts dieser Befunde ist es nicht verwunderlich, dass auch Wrede (1895) die $<$ g $>$-Aussprache als ein Beispiel anführt, um die ,richtige Interpretation der Sprachatlaskarten“ zu illustrieren: ${ }^{39}$

Regelmäßige $g$-Schreibung lässt durchaus nicht ohne weiteres auf Verschlusslaut schließen, beruht vielmehr oft darauf, dass die betreffende Mundart für das $g$ in allen Stellungen nur einen Laut besitzt, der auch beim Schriftdeutschsprechen gilt (mag es nun Spirans oder Explosiva sein), und daher keinen Grund zu unterscheidenden Schreibungen hatte.

(Wrede 1895, S. 41)

Belege für die auch über das 19. Jahrhundert hinaus währende Konstanz dieser Laut/Schriftzeichen-Zuordnung (Schriftzeichen $\langle\mathrm{g}\rangle=$ spirantische Aussprache) - auch über das Moselfränkische hinaus - finden wir (mindestens) bis zum Ende des 20. Jahrhunderts. So nehmen etwa Klein/Mattheier/ Mickartz (1978, S. 73-76) die ,Verwechslung von Rheinisch /j/,/r/, /sch/, /ch/ und Hochdeutsch /g/" in die Reihe der Interferenzfehler rheinischer Schüler auf, ${ }^{40}$ und auch das ,Wittlicher Wörterbuch“ ( $\left.{ }^{2} 1984\right)$ enthält laiensprachliche Einträge aus der Mitte des letzten Jahrhunderts (s. (7)), in denen eine Interpretation der spirantischen Aussprache von $<\mathrm{g}>$ außer Frage steht (s. beson$\operatorname{ders}(7 a)) .^{41}$

39 Für diesen Literaturhinweis danke ich herzlich Jürgen Erich Schmidt (Marburg).

40 Auch Stellmacher (1981, S. 63) zitiert „zahlreiche hyperkorrekte Schreibungen“ im Bereich der/g/-/x, c/-Alternation, die er als „Anzeichen für die große Unsicherheit in diesem Fehlerbereich “ niedersächsischer Schüler deutet: ,, Wir haben noch fröhlige Tage. [...] Weihnagsgeschenk. " $\mathrm{Zu}$ vergleichbaren Fehlern westfälischer bzw. hessischer Schüler s. Niebaum (1977, S. 47-50) bzw. Hasselberg/Wegera (1976, S. 40-42).

41 Zu einer ausführlicheren Analyse der Wörterbucheinträge s. Lenz (2003, S. 197-199). 
(7) Einträge aus dem „Wittlicher Wörterbuch“ ( $\left.{ }^{2} 1984\right)$

(7a)

\begin{tabular}{|c|c|c|}
\hline raße & $\begin{array}{l}\text { Basisdialekt } \\
{\left[\varepsilon: \beta_{1} \int \text { dg̊a:s] }\right.}\end{array}$ & $\begin{array}{l}\text { Wörterbucheinträge } \\
\text { Ewigdgaahs }\end{array}$ \\
\hline Wittlich & [vedilis] & Wedlig \\
\hline
\end{tabular}

(7b)

$\begin{array}{lll} & \text { Basisdialekt } & \text { Wörterbucheinträge } \\ \text { Waage } & {[\mathrm{vo:x]}} & \text { Woog } \\ \text { Tage } & {[\mathrm{d} \varepsilon: \mathrm{J}]} & \text { Dääg }\end{array}$

(7c)

$\begin{array}{lll} & \text { Basisdialekt } & \text { Wörterbucheinträge } \\ \text { genug } & {\left[\mathrm{g}^{\circ} \mathrm{no:x}\right]} & \text { genooch } \\ \text { Krieg } & {[\mathrm{kRe}: \mathfrak{f}]} & \text { Kreesch }\end{array}$

Zusammenfassend ist aus Beispiel 1 phänomenspezifisch abzuleiten: Auch in der Wenkerzeit sind die spirantischen Reflexe des westgermanischen $g$ nicht nur ein Charakteristikum der moselfränkischen Dialekte, sondern des gesamten mündlichen Spektrums moselfränkischer Sprecher. ${ }^{42}$ Bezüglich des Wenkermaterials ist generell abzulesen, dass sich aus den Dialektübersetzungen der Lehrer auch Aussagen über das Verhältnis von Laut und Schrift Ende des 19. Jahrhunderts und damit auch über die eigentlich von Wenker intendierte Varietät Dialekt hinaus, nämlich über das landschaftlich geprägte Hochdeutsch der Zeit, ablesen lassen. ${ }^{43}$ Im Blick auf das Gesamtmaterial stellen solche vermeintlichen „Fehler" der Lehrer den Ausnahmefall dar. Wie aktuelle und mit modernster Technik durchgeführte Analysen des Wenkermaterials belegen, können die Lehrertransliterationen insgesamt als verlässliche und exakte Datenquelle für die Dialekte Ende des 19. Jahrhunderts bewertet werden. $^{44}$

42 Nach Elspaß (2000, S. 268) „kann für das 19. Jh. wohl kaum von umgangssprachlichen Varietäten gesprochen werden, die den Sprecherinnen und Sprechern als Wahlmöglichkeiten zwischen Dialekt und Standard zur Verfügung standen. Für sie war ihr - aus heutiger normativer Sicht unzulängliches - in tendiertes Hochdeutsch die Form der für sie größtmöglichen Annäherung an den Standard.“

43 S. hierzu Herrgen/Schmidt (in Bearb.): „Im Rahmen der Sprachdynamikforschung beginnen wir soeben erst zu verstehen, dass uns dieser offensichtliche methodische Schwachpunkt [der Laienschreibungen im Wenkermaterial; A. N. L.] nicht nur die Möglichkeit beschert hat, den Dialektwandel anhand empirischer Daten über ein Jahrhundert hinweg zu verfolgen, sondern uns darüber hinaus ungeahnte Einblicke in das sprechsprachliche Gesamtsystem des Deutschen im ausgehenden 19. Jahrhundert ermöglicht."

S. etwa die Analysen von Herrgen (2006), Rabanus 2005 und Schmidt 2005. 


\section{Beispiel 2: Variationslinguistische Analyse auf der Grundlage von Zwirner- und Pfeffer-Korpus}

Die zweite Beispielanalyse will einen Einblick in die variationslinguistische Arbeit mit den regionalsprachlichen Tonkorpora der „Datenbank Gesprochenes Deutsch (DGD)" geben. Unter diesen sind bislang das Pfeffer-Korpus und das Zwirner-Korpus komplett bzw. zu größeren Teilen aufbereitet und zur online-Recherche freigegeben. ${ }^{45}$ Als exemplarischer Untersuchungsgegenstand, der auf der Grundlage dieser verfügbaren Daten analysiert werden soll, wurde das „Rezipientenpassivs“ ausgewählt. ${ }^{46}$ Dieses Phänomen steht neben anderen grammatischen und semantischen Besonderheiten, die das verbale Wortfeld des Gebens und Bekommens im Deutschen, aber auch in anderen, besonders westgermanischen Sprachen aufweist (s. Lenz 2007a).

\section{(8) Aktiv, Vorgangs- und Rezipientenpassiv im Vergleich}

\section{Aktiv Sein Vater überreichte ihm das Geschenk. \\ Vorgangspassiv Das Geschenk wurde ihm (von seinem Vater) überreicht. \\ Rezipientenpassiv Er bekam das Geschenk (von seinem Vater) überreicht.}

Wie die Bezeichnung ausdrückt, ist es (häufig) der Rezipient der Handlung, der im Rezipientenpassiv fokussiert wird, indem ihm Subjektstatus zukommt. Als Auxiliare des Rezipientenpassivs dienen bekommen und kriegen (seltener auch erhalten). Dabei fungiert bekommen (bzw. erhalten) als stilistisch gehobene und schriftsprachliche Variante, während kriegen als Variante des mündlichen Registers bewertet wird (vgl. Duden ${ }^{72005}$, S. 557). Was die dialektale Verbreitung des Rezipientenpassivs im Deutschen angeht, hat erstmals und jüngst Glaser (2005) detailliertere Ergebnisse formuliert. Ihre Auswertung der Forschungsliteratur bzw. der Daten aus dem Syntaxatlas der Schweiz ergibt,

dass das Mitteldeutsche zweifellos als Kernlandschaft des ,bekommen'-Passivs angesprochen werden kann, während es sich für das Niederdeutsche zwar ebenfalls sehr weiträumig nachweisen lässt, sich aber dennoch der Eindruck ergibt, dass es keinesfalls die gleiche Produktivität und Vielfältigkeit besitzt [...]. Die Untersuchung zum Schweizerdeutschen hat [...] ergeben, dass es allenfalls Ansätze zur Herausbildung eines , bekommen'-Passivs gibt, dass dieses aber mit grosser Wahrscheinlichkeit keine dialektale Tradition hat.

(Glaser 2005, S. 50 u. 54)

45 Das TAVM-Korpus bleibt in der folgenden Analyse unberücksichtigt, da erstens bislang nur ca. ein Drittel der Tonaufnahmen/Transkripte zur Recherche freigegeben sind und zweitens diese aufbereiteten Daten fast ausschließlich zum Schlesischen gehören. Hinzukommt, dass das TAVM-Korpus aufgrund der methodischen Anlage der Datenerhebung deutlich basisdialektaler einzustufen ist als das Zwirner-Korpus. Insofern verfälschte eine quantitative Analyse auf der Grundlage der vereinten Zwirner- und TAVM-Daten das Gesamtbild.

46 Zum Phänomen im Deutschen s. Leirbukt 1997, Glaser 2005, Duden ( ${ }^{7} 2005$, S. 556558) und Zifonun [u.a.] (1997, S. 1824-1829) und Lenz 2007b. 
Diese Thesen bezüglich der Arealität des Rezipientenpassivs werden im Folgenden auf der Grundlage der in der „Datenbank Gesprochenes Deutsch (DGD)“ verfügbaren regionalsprachlichen Tonaufnahmen aus dem Zwirnerund dem Pfeffer-Korpus überprüft.

In (9) sind erste Ergebnisse der Korpusrecherchen einzusehen. Die Analyse aller bekommen-, kriegen- und erhalten-Belege, die in den 2430 alignierten Transkripten der beiden Korpora ermittelt werden konnten, ergibt insgesamt 432 Rezipientenpassiv-Belege, was einem prozentualen Anteil von 17,8\% entspricht. Das bedeutet, dass in jeder fünften bis sechsten verfügbaren Tonaufnahme ein Beleg für ein Rezipientenpassiv zu finden ist. Die Tatsache, dass die Aufnahmen aus Zwirner- und Pfeffer-Korpus jeweils ca. 12 Minuten Aufnahmelänge umfassen und damit eine vergleichbare Dauer aufweisen, stützt die Aussagekraft der berechneten Prozentzahlen. (Genauere Angaben bezüglich des Wort- bzw. Satzumfangs der Teilkorpora, die der Recherche zugrunde lagen, können hier nicht gemacht werden, da diese Recherchefunktion in der DGD noch nicht möglich ist. ${ }^{47}$ )

(9) Rezipientenpassive im Zwirner- und Pfeffer-Korpus

\begin{tabular}{l|c|c|c} 
& \multirow{2}{*}{$\begin{array}{l}\text { Anzahl der } \\
\text { verfügbaren }\end{array}$} & \multicolumn{2}{|l}{ Rezipientenpassive } \\
\cline { 3 - 4 } & Tonaufnahmen & absolute Häufigkeiten & relative Häufigkeiten \\
\hline Zwirner & 2032 & 360 & $\mathbf{1 7 , 7} \%$ \\
Pfeffer & 398 & 72 & $\mathbf{1 8 , 1} \%$ \\
\hline GESAMT & $\mathbf{2 4 3 0}$ & $\mathbf{4 3 2}$ & $\mathbf{1 7 , 8} \%$
\end{tabular}

Als Rezipientenpassive wurden - in Anlehnung an Leirbukt (1997) - nur solche Konstruktionen bestehend aus „,bekommen/erhalten/kriegen + Part. II“ gewertet, die eine Retransformierung des Passivsatz-Subjekts in einen Aktivsatz-Dativ (Dativobjekt oder auch frei(er)er Dativ) ermöglichen. Folglich wurden die in (10) aufgeführten Konstruktionen nicht als Rezipientenpassive gezählt. Während das Partizip gehackt in (10a) (aus einer Aufnahme des TAVM-Korpus) als Objektsprädikativ in Verbindung mit dem Vollverb kriegen im Sinne von, erhalten, empfangen' zu interpretieren ist und somit keine Verbalperiphrase vorliegt, exemplifiziert (10b) eine kriegen-Periphrase zum

47 In den Korpusinformationen der DGD finden sich aktuell lediglich Angaben über den digitalisierten Gesamtumfang eines Korpus, unabhängig von seiner Verfügbarkeit und damit auch unabhängig von seiner Recherchierbarkeit im Internet (http://dsav-wiss.idsmannheim.de/DSAv/KORPORA/). Angaben über Teilbestände, die einer Recherche zugrunde liegen (z. B. Wortumfang der alignierten Transkripte eines Teilkorpus zu einer ausgewählten „Mundartgruppe“) können noch nicht erfragt werden. Da das PfefferKorpus komplett aufbereitet und online gestellt ist, liegt das gesamte digitalisierte Korpus auch der Berechnung in (9) zugrunde. Das Pfeffer-Korpus umfasst insgesamt 645.540 Wörter (tokens), darunter 42.075 unterschiedliche Wortformen (types). 
Ausdruck des Bewerkstelligens. Bei dieser Modalkonstruktion handelt es sich nach Glaser (2005, S. 60) dialektologisch gesehen wohl „um eine im wesentlichen niederdeutsche (und niederfränkische)" Konstruktion.

Zumindest im Westen reicht die Konstruktion auch in den hochdeutschen Raum hinein, worauf Belege aus dem Luxemburgischen [...] und dem Ripuarischen deuten. [...] Im Unterschied zum ,bekommen'-Passiv, das auch schriftlich gut und v. a. auch räumlich breit belegt ist, ist das modale Aktiv mit kriegen/bekommen - erhalten kommt hier nicht in Frage - aber offenbar bislang weitgehend an die gesprochene Sprache gebunden geblieben und hat auch dabei (noch) keine überregionale Verbreitung gefunden. Es ist zu vermuten, dass es aus den niederdeutschen Basisdialekten, wo es sich sogar wesentlich besser als das ,bekommen'-Passiv belegen lässt, in den (nördlich geprägten) regionalen Substandard eingedrungen ist.

(Glaser 2005, S. 59)

Die dritte, in (10c) exemplifizierte Konstruktion, die nicht in die Berechnung der Rezipientenpassivbelege eingegangen ist, weist ein Subjekt auf, das mit einem Aktivsatz-Akkusativ korrespondiert, bzw. umfasst Sätze, bei denen nicht zwangsweise von einer Ellipse eines Akkusativobjektes ausgegangen werden kann. ${ }^{48}$

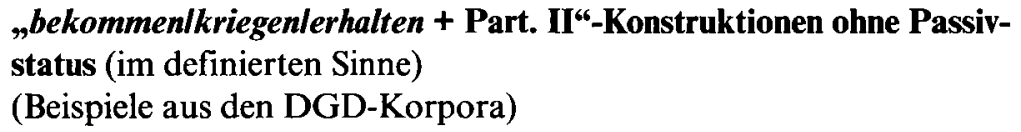

„bekommenlkriegenlerhalten + Part. II“-Konstruktionen ohne Passivstatus (im definierten Sinne) (Beispiele aus den DGD-Korpora)

(10a) Objektsprädikativ mit Zustandsinterpretation

OS 494: [...] dann kriegten sie auch ein gekochtes Hühnerei fein gehackt ,dann bekamen sie auch ein fein gehacktes gekochtes Hühnerei $^{6}$

(10b) Objektsprädikativ mit kausativ-resultativer Interpretation („Modalkonstruktion“)

ZW8L7: [...] da haben wir so Mühe mit, dass wir das dann getan kriegen ,wir haben Mühe damit, es zu schaffen`

(10c) Aktivsatz-Akkusativ

ZWY08: [...] und deshalb hab ich auch oft geschimpft gekriegt ,und deshalb bin ich auch oft geschimpft worden"

Der in (9) ermittelte korpusübergreifende Gesamtwert von 17,8\% weicht nur minimal von den ermittelten Frequenzen des Rezipientenpassivs in den beiden Teilkorpora ab (17,7\% bzw. 18,1\%). Obwohl das Zwirner-Korpus insgesamt dialektalere Aufnahmen als das „umgangssprachlichere“" Pfeffer-Korpus umfasst, zeigen die beiden Korpora hinsichtlich des Rezipientenpassivs

48 Von der Ellipse eines Akkusativobjektes wurde dagegen in Konstruktionen mit bezahlen oder verhauen $\mathrm{u}$. a. ausgegangen. Eine ausführlichere Diskussion des in (10c) exemplifizierten Konstruktionstyps kann hier nicht geleistet werden. S. dazu Leirbukt (1997, S. 13-15). Zur Klassifikation der Konstruktionen in (10) siehe auch Zifonun [u.a.] (1997, S. 1827). 
keine signifikanten quantitativen Unterschiede. Differenzen zwischen den beiden Korpora werden jedoch offensichtlich, wenn nach den konkreten Auxiliaren gefragt wird, die in den Rezipientenpassivbelegen vorkommen. Wie aus Tabelle (11) abzulesen ist, wird das Rezipientenpassiv im Pfeffer-Korpus zu $79,2 \%$ mit dem Auxiliar bekommen realisiert, wohingegen im Zwirner-Korpus kriegen mit 90,3\% eindeutig als Auxiliar überwiegt. Erhalten ist mit nur einem Beleg im Pfeffer-Korpus vertreten. Wenn auch Zwirner- und PfefferKorpus hinsichtlich der Quantität realisierter Rezipientenpassive kaum differieren, sind sie somit in der Qualität der Belege klar voneinander abzugrenzen. Die dialektalere Anlage der Zwirner-Aufnahmen kommt bezüglich des Rezipientenpassivs in der Dominanz des Passivauxiliars kriegen zum Ausdruck, während in den standardsprachnäheren Pfeffer-Aufnahmen das Auxiliar bekommen dominiert.

(11) Auxiliare des Rezipientenpassivs im Zwirner- und Pfeffer-Korpus (absolute und relative Häufigkeiten)

\begin{tabular}{c|cc|cc|cc} 
& \multicolumn{2}{|c|}{ kriegen } & \multicolumn{2}{c|}{ bekommen } & \multicolumn{2}{c}{ erhalten } \\
\hline Zwirner & 325 & $\mathbf{9 0 , 3} \%$ & 35 & $\mathbf{9 , 7} \%$ & - & - \\
Pfeffer & 14 & $\mathbf{1 9 , 4} \%$ & 57 & $\mathbf{7 9 , 2} \%$ & 1 & $\mathbf{1 , 4} \%$ \\
\hline GESAMT & 339 & $\mathbf{7 8 , 5} \%$ & 92 & $\mathbf{2 1 , 3} \%$ & 1 & $\mathbf{0 , 2} \%$
\end{tabular}

Aus den Frequenzwerten in (9) könnte die These abgeleitet werden, dass sich das Rezipientenpassiv - zumindest hinsichtlich seiner Vorkommenshäufigkeit - relativ gleichmäßig über die Dialekte (annähernd repräsentiert im ZwirnerKorpus) und Regiolekte (annähernd repräsentiert im Pfeffer-Korpus) des Deutschen verteilt. Um diese These zu verifizieren, ist ein Blick auf die areale Verteilung der Belege notwendig, die in (12) - zunächst für Zwirner- und Pfeffer-Korpus gemeinsam - einzusehen ist. Das Balkendiagramm zeigt die relativen Häufigkeiten von Rezipientenpassiven in ihrer Verteilung auf die „Mundartgruppen“, wie sie in der DGD-Abfrage aufgeführt sind. Unberücksichtigt blieben die Mundartgruppen Friesisch, Mecklenburgisch-Vorpommersch, Märkisch, Schleswigsch, Westthüringisch und Südbairisch, da die Anzahl ihrer verfügbaren Aufnahmen deutlich unter dem Durchschnitt von ca. 108 Aufnahmen pro Mundartgruppe liegen, sowie die Aufnahmen aus „außereuropäischen Sprachinseln“. 


\section{Areale Verteilung der Rezipientenpassive aus} Zwirner- und Pfeffer-Korpus

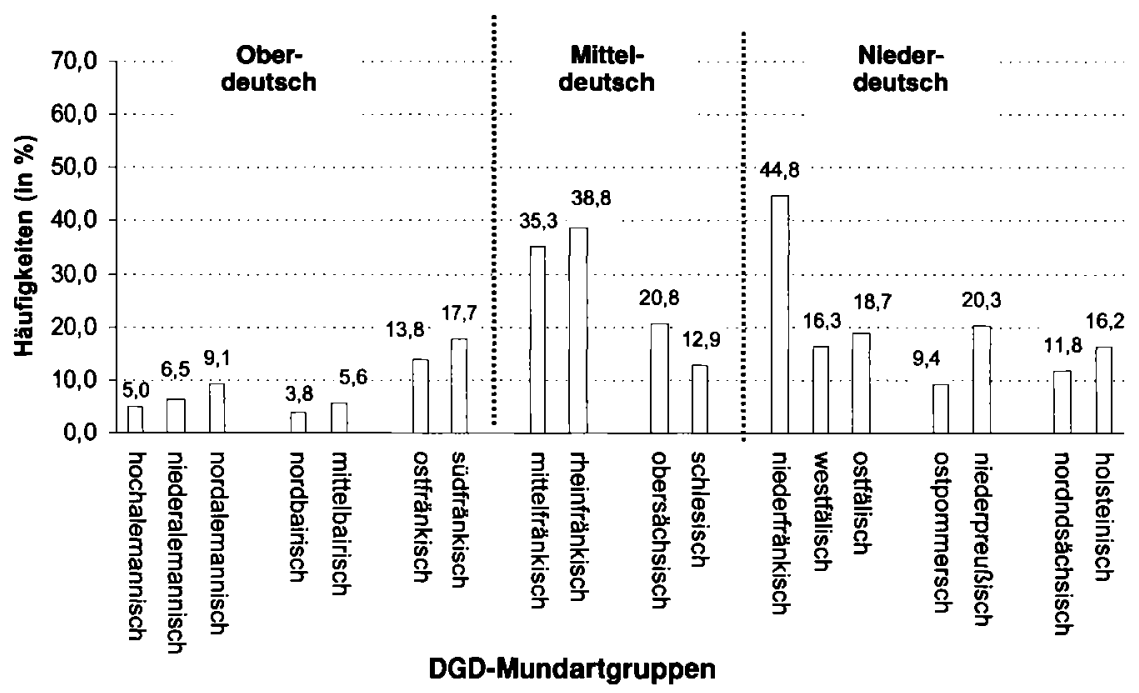

Das Rezipientenpassiv ist in allen hier differenzierten Regionen des deutschsprachigen Raums vertreten, allerdings in deutlich unterschiedlichen Frequenzen. Unter den DGD-Mundartgruppen zeigen das Niederfränkische $(44,8 \%)$, das Mittelfränkische (35,3\%) und das Rheinfränkische $(38,8 \%)$ die höchsten Frequenzwerte, wobei zu bedenken ist, dass auch die Großgruppe der hessischen Aufnahmen in die DGD-Kategorie "Rheinfränkisch" eingeordnet ist.

Auf einer höheren Klassifizierungsebene sind die Mundartgruppen den drei großen Sprachräumen Nieder-, Mittel- und Oberdeutsch zugeordnet. Wie aus der Zusammenschau von Balkenhöhe und Sprachraum ablesbar wird, weist das Mitteldeutsche auch insgesamt die höchsten Frequenzen an Rezipientenpassiven auf, nämlich insgesamt $33,6 \%$, gefolgt vom Niederdeutschen mit insgesamt 18,5\%. Die geringste Anzahl an Rezipientenpassiven ist im oberdeutschen Raum zu finden $(8,4 \%)$. Innerhalb der nieder- und oberdeutschen Mundarten sind es jeweils die an das Westmitteldeutsche angrenzenden Mundarten, die die höchsten Frequenzen an Rezipientenpassive innerhalb ihres Großraumes aufweisen, nämlich das Niederfränkische im Niederdeutschen bzw. das Süd- und Ostfränkische im Oberdeutschen.

In (13) und (14) sind die Frequenzen des Rezipientenpassivs für Zwirnerund Pfeffer-Korpus getrennt aufgeführt. Es fanden jeweils nur solche Mundartgruppen Berücksichtigung, die mit mehr als $10 \mathrm{bzw}$. 20 recherchierbaren Aufnahmen im Pfeffer- bzw. Zwirner-Korpus vertreten sind. 
(13) Areale Verteilung der Rezipientenpassive im Zwirner-Korpus

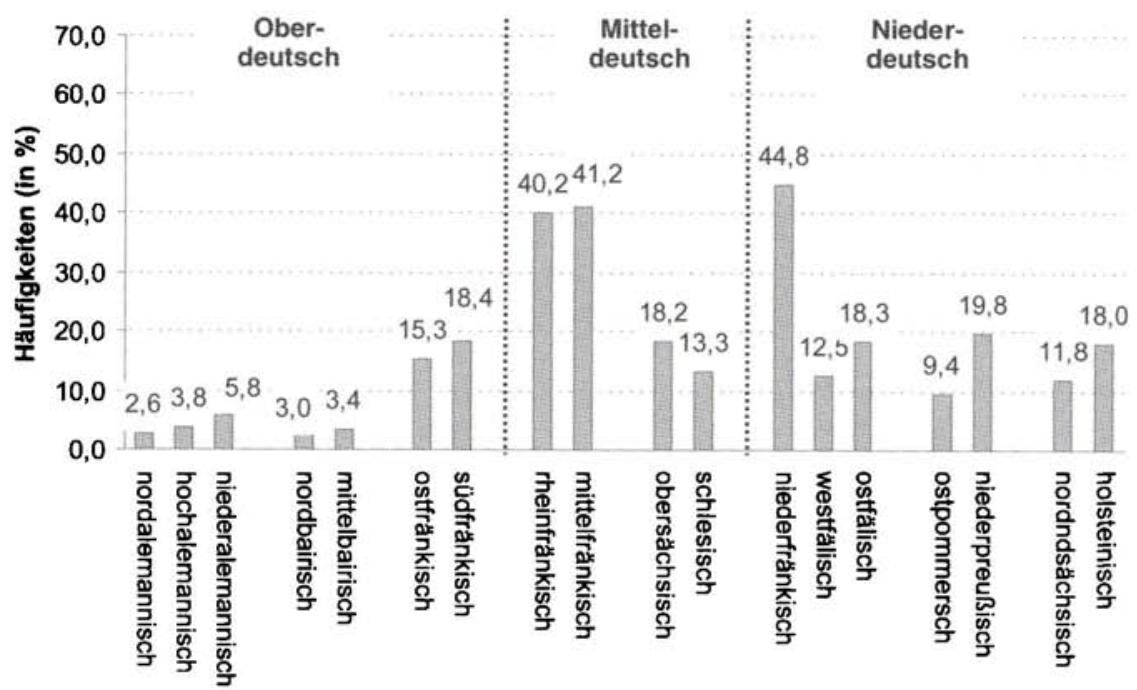

DGD-Mundartgruppen

Aus dem Vergleich der dialektaleren Zwirner- mit den standardnäheren Pfeffer-Aufnahmen lassen sich folgende Thesen ableiten: Das Rezipientenpassiv ist ein genuin dialektales Phänomen des Westmitteldeutschen und - mit Blick auf Abbildung (13) - des nordwestlich angrenzenden Niederfränkischen. ${ }^{49}$ In den Zwirner-Aufnahmen dieser Regionen tritt das Rezipientenpassiv in Frequenzen von über $40 \%$ auf, während die Vorkommenshäufigkeiten im Zwirner-Korpus für alle anderen Regionen bei unter $20 \%$ liegen. Ausgehend von diesem Ergebnis werden die drei benachbarten Regionen Niederfränkisch, Mittelfränkisch und Rheinfränkisch als dialektaler Kernraum des Rezipientenpassivs ausgewiesen. Von diesem Zentrum breitet sich das Phänomen sukzessive sowohl horizontal (in der Fläche) als auch vertikal (auf der DialektStandard-Achse) in die Regionalsprachen des Deutschen aus. Dabei ist die Progression des Rezipientenpassivs in den 50ern und 60ern des letzten Jahrhunderts, in denen die Zwirner- und Pfeffer-Aufnahmen entstanden sind, im Vergleich der Graphiken (13) und (14) besonders im Westniederdeutschen und im Oberdeutschen (bes. nordalemannisch und mittelbairisch) nachzuvollziehen.

49 Da das Pfeffer-Korpus keine Aufnahmen aus dem Niederfränkischen enthält, musste diese Dialektregion in Abbildung (14) unberücksichtigt bleiben. Die hohe Frequenz an Rezipientenpassivbelegen, die für das Niederfränkische aus Abbildung (12) abzulesen ist, basiert somit allein auf Zwirner-Aufnahmen. 


\section{(14) Areale Verteilung der Rezipientenpassive im Pfeffer-Korpus}

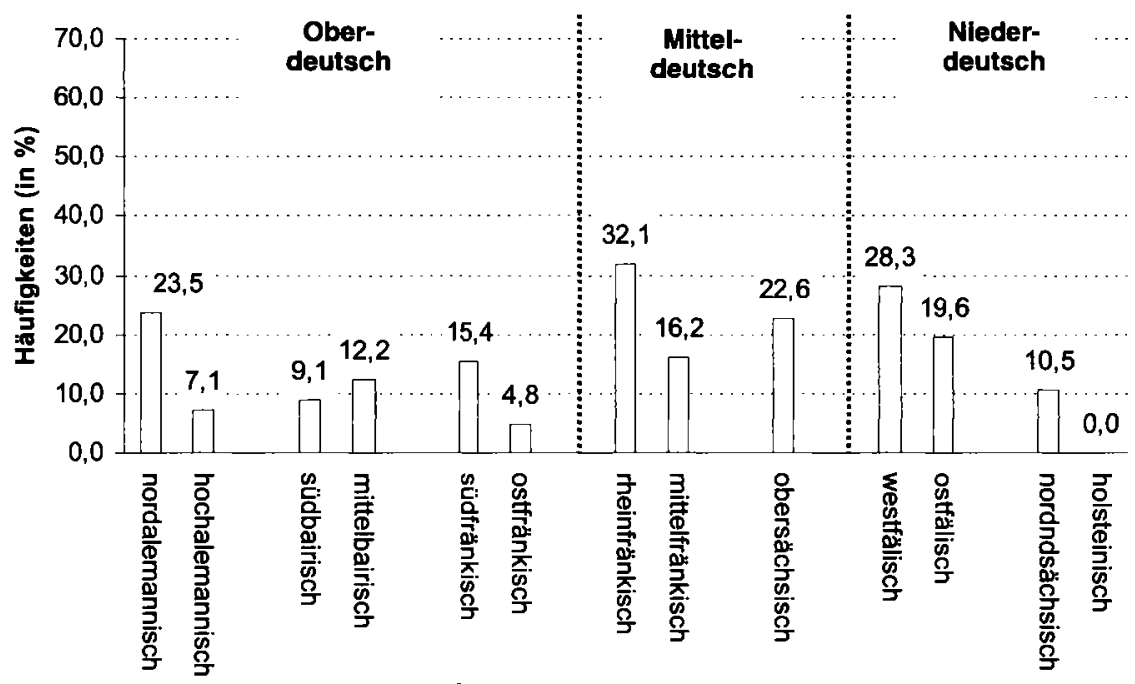

DGD-Mundartgruppen

Unter den oberdeutschen Regionen ist es das Bairische, das insgesamt die niedrigsten Frequenzen an Rezipientenpassiven aufweist (s. (12)). Für diese Region können aus dem Vergleich von (13) und (14) Evidenzen für die von Glaser (2005) aufgestellte These abgeleitet werden:

[...] Unsicherheiten bestehen bezüglich der Verhältnisse im Bairischen, wo bislang nur für das ans Mitteldeutsche grenzende Egerländische der Gebrauch verschiedener Passivperiphrasen belegt ist, während im übrigen die Konstruktionsmöglichkeiten sehr restringiert zu sein scheinen.

(Glaser 2005, S. 54)

Ebenso wird Glasers These (2005, S. 54), dass das Rezipientenpassiv im Schweizerdeutschen „mit grosser Wahrscheinlichkeit keine dialektale Tradition hat", durch die nördlich ans Höchstalemannisch anschließenden Mundartgruppen Hochalemannisch und Nordalemannisch gestützt. Denn in diesen beiden Regionen - wie auch im Bairischen - sind es die Pfeffer- und damit die dialektferneren Aufnahmen, die eine höhere Frequenz an Rezipientenpassiven aufweisen als die Zwirner-Aufnahmen.

Phänomenspezifisch ist aus der zweiten Beispielanalyse abzuleiten: Die Ergebnisse, die aus der Analyse von Zwirner- und Pfeffer-Korpus gewonnen wurden, stützen nicht nur die Thesen, die auf der Grundlage anderer Datenquellen formuliert wurden, sondern die Korpusdaten ermöglichen auch einen tieferen Einblick in die regionalsprachlichen Verhältnisse des Rezipientenpassivs. Generell ist bezüglich der variationslinguistischen Arbeit mit den DGDKorpora festzuhalten: Auch wenn es sich (bislang) bei den recherchierbaren Transkripten um reine Textdateien ohne linguistische Annotationen handelt, 
können das Zwirner- und Pfeffer-Korpus bereits in ihrem aktuellen Bearbeitungsstand als eine höchst ergiebige Analysequelle für die Regionalsprachen der zweiten Hälfte des 20. Jahrhunderts bewertet werden. Wie am Beispiel des Rezipientenpassivs exemplifiziert werden konnte, repräsentiert das ZwirnerKorpus einen „tieferen“, das heißt dialektaleren regionalsprachlichen Spektrumsbereich als das standardsprachnähere Pfeffer-Korpus.

\section{Zusammenfassung}

Im Zentrum des Beitrags standen korpuslinguistische Fragestellungen aus variationslinguistischer Perspektive. Dabei lag der Fokus auf der Analyse regionalsprachlicher Daten bzw. Korpora.

Im Rahmen einer Überblicksdarstellung wurden zunächst Datenklassen präsentiert, die zu regionalsprachlichen Analysen herangezogen werden (können). Unter den beiden Hauptklassen „(objektive) Sprachdaten“ und ,(subjektive) Spracheinstellungsdaten“" wurden erstere als Diskussionsgrundlage für diesen Beitrag ausgewählt. Zur weiteren Klassifikation der Sprachdaten diente - in Anlehnung an das Konzept von Koch/Oesterreicher (1985), (1994) - das Medium der sprachlichen Äußerung als zentrales Klassifikationskriterium. Wie ein Blick in die Regionalsprachenforschung zeigt, überwiegen sowohl unter den medial mündlichen als auch unter den medial schriftlichen Daten elizitierte Sprachdaten, die mit unterschiedlichsten variationslinguistischen Methoden der Datenerhebung gewonnen werden.

Vor dem Hintergrund der Beliebtheit und Ergiebigkeit elizitierter Sprachdaten für regionalsprachliche Fragestellungen konzentrierte sich die anschlieBende Präsentation regionalsprachlicher Korpora des Deutschen auf exemplarische Sprachkorpora, die auf elizitierten Daten aufbauen. Angelehnt an den allgemeinen Korpusbegriff von McEnery/Wilson (1996) wurden einem regionalsprachlichen Korpus folgende Merkmale zugeschrieben:

- Es handelt sich um eine abgeschlossene Menge an medial mündlichen oder medial schriftlichen Sprachdaten.

- Das Korpus ist für die modernen Regionalsprachen des Deutschen bzw. für bestimmte regionalsprachliche Spektrumsausschnitte (Varietäten) repräsentativ.

- Das Korpus ist durch Meta-Beschreibungen erschlossen.

- Das Korpus ist (im weiteren Sinne) computertechnisch aufbereitet.

Als ein Beispiel regionalsprachlicher Korpora, die auf elizitierten medial schriftlichen Sprachdaten basieren, diente das Wenker-Korpus, das im „Digitalen Wenker-Atlas (DiWA)“ online verfügbar ist. Als Untersuchungsgegenstand, an dem die Spezifik und Ergiebigkeit dieses Korpus exemplifiziert wurde, fungierte die Plosiv-Spirans-Variation im Bereich des Schriftzeichens $\langle\mathrm{g}\rangle$. Als Beispiele medial mündlicher elizitierter Sprachkorpora wurden vier Tonkorpora der „Datenbank Gesprochenes Deutsch (DGD)“ vorgestellt. 
Unter diesen wurden das Zwirner- und das Pfeffer-Korpus im Rahmen einer Analyse zum Rezipientenpassiv auf ihre Analysemöglichkeiten und ihre Ergiebigkeit untersucht.

In den Beispielanalysen wurde offensichtlich, dass sowohl das Wenker-Korpus als auch das Zwirner- und Pfeffer-Korpus hinsichtlich des analysepraktischen als auch bezüglich des zu leistenden Interpretationsaufwands sicherlich keine „einfachen“ Korpora darstellen. Zum Beispiel liegen den drei Korpora keine linguistischen Annotationen zugrunde, die Voraussetzung für unkomplizierte(re) Abfragemöglichkeiten darstellen würden. Im Falle des WenkerKorpus kommt hinzu, dass aufgrund der Kurrentschrift eine automatische Erfassung der Wenkerbogen als Textdatei (bislang) noch nicht möglich ist. Trotz der skizzierten Probleme, die die Korpora aus korpuslinguistischer Sicht aufweisen, konnten die vorgestellten Analysen die hohe Ergiebigkeit und Validität der ausgewählten Korpora nachweisen. Vor dem Hintergrund der skizzierten Ergebnisse bleibt auf weitere Erschließung der Materialien in naher Zukunft zu hoffen.

\section{Literatur}

Ágel, Vilmos/Hennig, Mathilde (2006): Theorie des Nähe- und Distanzsprechens. In: Ágel, Vilmos/Hennig, Mathilde (Hg.) (2006): Grammatik aus Nähe und Distanz. Theorie und Praxis am Beispiel von Nähetexten 1650-2000. Tübingen: Niemeyer. S. 3-31.

Ammon, Ulrich [u.a.] (Hg.) (2005): Sociolinguistics/Soziolinguistik. Ein internationales Handbuch zur Wissenschaft von Sprache und Gesellschaft. 2., vollständig neu bearbeitete und erweiterte Aufl. Berlin/New York: Walter de Gruyter (Handbücher zur Sprach- und Kommunikationswissenschaft 3.2).

Androutsopoulos, Jannis/Schmidt, Gurly (2002): SMS-Kommunikation: Ethnografische Gattungsanalyse am Beispiel einer Kleingruppe. In: Zeitschrift für Angewandte Linguistik 36, S. 49-80.

Androutsopoulos, Jannis/Ziegler, Evelyn (2003): Sprachvariation und Internet: Regionalismen in einer Chat-Gemeinschaft. In: Androutsopoulos, Jannis/Ziegler, Evelyn (2003): „Standardfragen“. Soziolinguistische Perspektiven auf Sprachgeschichte, Sprachkontakt und Sprachvariation. Frankfurt/M. [u.a.]: Peter Lang (VarioLingua 18). S. 251-279.

Aschwanden, Brigitte (2001): „Wär wot chätä?“ Zum Sprachverhalten deutschschweizerischer Chatter. Networx 24 [http://www.mediensprache.net/de/networx/networx-24. pdf].

Atteslander, Peter (2005): Schriftliche Befragung/Written Investigations. In: Ammon, Ulrich [u. a.] (Hg.), S. 1063-1076.

Auer, Peter (1995): Ethnographic Methods in the Analysis of Oral Communication. Some Suggestions for Linguists. In: Quasthoff, Uta M. (ed.): Aspects of Oral Communication. Berlin/New York: Walter de Gruyter. S. 419-440.

Auer, Peter/Hinskens, Frans (1996): The Convergence and Divergence of Dialects in Europe. New and not so New Developments in an Old Area. In: Auer, Peter [u. a.] (Hg.): Konvergenz und Divergenz von Dialekten in Europa. Tübingen: Niemeyer (Sociolinguistica 10). S. 1-30.

Auwärter, Manfred (2005): Experiment/Experiments. In: Ammon, Ulrich [u.a.] (Hg.), S. $1076-1084$. 
Beißwenger, Michael (Hg.) (2001): Chat-Kommunikation. Sprache, Interaktion, Sozialität \& Identität in synchroner computervermittelter Kommunikation. Perspektiven auf ein interdisziplinäres Forschungsfeld. Stuttgart: Ibidem.

Bellmann, Günter (1964): Wege und Möglichkeiten der Schallaufnahme ostdeutscher Mundarten heute. Zur Tonbandaufnahme der Vertriebenenmundarten. In: Zeitschrift für Mundartforschung 31, S. 62-79.

Bellmann, Günter (1970): Einleitung. In: Bellmann, Günter/Göschel, Joachim: Tonbandaufnahme ostdeutscher Mundarten 1962-1965. Gesamtkatalog. Marburg: Elwert (Deutsche Dialektgeographie 73). S. 7-30.

Bellmann, Günter (1983): Probleme des Substandards im Deutschen. In: Mattheier, Klaus J. (Hg.): Aspekte der Dialekttheorie. Tübingen: Niemeyer (Reihe Germanistische Linguistik 46). S. 105-130.

Bellmann, Günter (1994): Einführung in den Mittelrheinischen Sprachatlas (MRhSA) von Günter Bellmann. Tübingen: Niemeyer.

Bellmann, Günter/Göschel, Joachim (1970): Tonbandaufnahme ostdeutscher Mundarten 1962-1965. Gesamtkatalog. Marburg: Elwert (Deutsche Dialektgeographie 73).

Bellmann, Günter/Herrgen, Joachim/Schmidt, Jürgen Erich (1994-2002): Mittelrheinischer Sprachatlas (MRhSA). Bd. 1-5. Tübingen: Niemeyer.

Besch, Werner [u. a.] (Hg.) (1983): Dialektologie. Ein Handbuch zur deutschen und allgemeinen Dialektforschung. Erster Halbbd. Berlin/New York: de Gruyter (Handbücher zur Sprach- und Kommunikationswissenschaft 1.1).

Briggs, Charles L. (2005): Sociolinguistic Interviews/Soziolinguistisches Interview. In: Ammon, Ulrich [u. a.] (Hg.), S. 1052-1062.

Bucheli, Claudia/Glaser, Elvira (2002): The Syntactic Atlas of Swiss German Dialects: emEmpirical and Methodological Problems. In: Barbiers, Sjef [et al.] (eds.): Syntactic Microvariation. [http://www.meertens.knaw.nl/books/synmic/]

Burger, Harald ( ${ }^{3} 2005$ ): Mediensprache. Eine Einführung in Sprache und Kommunikationsformen der Massenmedien. Berlin: de Gruyter.

Christen, Helen (2002): „Swiss Date“ - Aspekte dialektalen Sprechens im Schweizer Privatfernsehen. In: Wiesinger, Peter ( $\mathrm{Hg}$.): Akten des X. Internationalen Germanistenkongresses Wien 2000 „Zeitenwende - Die Germanistik auf. dem Weg vom 20. ins 21. Jahrhundert“. Bd. 3: Aufgaben einer zukünftigen Sprachgeschichtsforschung Gesprochene Sprache in regionaler und sozialer Differenzierung - Sprache in der Öffentlichkeit. Bern [u. a.]: Peter Lang. S. 217-225.

Christen, Helen/Tophinke, Doris/Ziegler, Evelyn (2005): Chat und regionale Identität. In: Krämer-Neubert, Sabine/Wolf, Norbert Richard (Hg.): Bayerische Dialektologie. Akten der Internationalen Dialektologischen Konferenz, 26.-28. Februar 2002. Heidelberg: Winter (Schriften zum Bayerischen Sprachatlas 8). S. 425-439.

Cornelissen, Georg/Schmitt, Eva-Maria (Hg.) (1997): Dialekte und Dialektliteratur in der Euregio Rhein-Maas-Nord. Unter Mitarbeit von Pierre Bakkes. Mit CD. Akustische Dokumentation Hans-Peter Beyenburg. Köln: Rheinland-Verlag.

Cornips, Leonie/Poletto, Cecilia (2005): On standardising syntactic elicitation techniques. Part I. In: Lingua 115 (7), S. 939-957.

DiWA $=$ Schmidt/Herrgen $2001 \mathrm{ff}$.

Duden ( ${ }^{4} 2000$ ): Aussprachewörterbuch. Wörterbuch der deutschen Standardaussprache. 4., neu bearbeitete und aktualisierte Auflage. Bearbeitet von Max Mangold in Zusammenarbeit mit der Dudenredaktion. Duden Band 6. Mannheim [u. a.]: Dudenverlag.

Duden ( $\left.{ }^{7} 2005\right)$ : Die Grammatik. Unentbehrlich für richtiges Deutsch. 7., völlig neu erarbeitete und erweiterte Auflage. Herausgegeben von der Dudenredaktion. Duden Band 4. Mannheim [u. a.]: Dudenverlag. 
Dürscheid, Christa (1999): Zwischen Mündlichkeit und Schriftlichkeit: die Kommunikation im Internet. In: Papiere zur Linguistik 60/1, S. 17-30.

Dürscheid, Christa (2002): E-Mail und SMS - ein Vergleich. In: Ziegler, Arne/Dürscheid, Christa (Hg.): Kommunikationsform E-Mail. Tübingen: Stauffenberg. S. 93-114.

Dürscheid, Christa (2005): Medien, Kommunikationsformen, kommunikative Gattungen. In: Linguistik online 22/1.

Eggers, Eckhard/Schmidt, Jürgen Erich/Stellmacher, Dieter (Hg.) (2005): Moderne Dialekte - Neue Dialektologie. Akten des 1. Kongresses der Internationalen Gesellschaft für Dialektologie des Deutschen (IGDD) am Forschungsinstitut für deutsche Sprache „Deutscher Sprachatlas“ der Philipps-Universität Marburg vom 5.-8. März 2003. Stuttgart: Steiner (Zeitschrift für Dialektologie und Linguistik. Beihefte 130).

Elspaß, Stephan (2000): Rheinische Sprachgeschichte von 1700-1900. In: Macha, Jürgen [u.a.] (Hg.): Rheinisch-westfälische Sprachgeschichte. Köln [u.a.]: Böhlau (Niederdeutsche Studien 46). S. 247-276.

Elspaß, Stephan (2005a): Sprachgeschichte von unten. Untersuchungen zum geschriebenen Altagsdeutsch im 19. Jahrhundert. Tübingen: Niemeyer (Reihe Germanistische Linguistik 263).

Elspaß, Stephan (2005b): Zum Wandel im Gebrauch regionalsprachlicher Lexik. Ergebnisse einer Neuerhebung. In: Zeitschrift für Dialektologie und Linguistik 72, S. 151 .

Fiehler, Reinhard/Wagener, Peter (2005): Die Datenbank Gesprochenes Deutsch (DGD) Sammlung, Dokumentation, Archivierung und Untersuchung gesprochener Sprache als Aufgaben der Sprachwissenschaft. In: Gesprächsforschung - Online-Zeitschrift zur verbalen Interaktion. Ausgabe 6, 136-147. [http://www.gespraechsforschungozs.de]

Glas, Reinhold/Ehlich, Konrad (2000): Deutsche Transkripte 1950 bis 1995. Ein Repertorium. Hamburg (Arbeiten zur Mehrsprachigkeit. Folge A. 63).

Glaser, Elvira (2000): Erhebungsmethoden dialektaler Syntax. In: Stellmacher, Dieter (Hg.): Dialektologie zwischen Tradition und Neuansätzen. Beiträge der internationalen Dialektologentagung, Göttingen, 19.-21. Oktober 1998. Stuttgart: Steiner (Zeitschrift für Dialektologie und Linguistik. Beihefte 109). S. 258-276.

Glaser, Elvira (2005): Krieg und kriegen: zur Arealität der BEKOMMEN-Periphrasen. In: Kleinberger Günther, Ulla [u.a.] (Hg.): „Krieg und Frieden“- Auseinandersetzung und Versöhnung in Diskursen. Tübingen: Francke. S. 43-64.

Günther, Ulla/Wyss, Eva Lia (1996): E-Mail-Briefe - Eine neue Textsorte zwischen Mündlichkeit und Schriftlichkeit. In: Hess-Lüttich, Ernest W. B. [u. a.] (Hg.): Textstrukturen im Medienwandel. Frankfurt/M. [u. a.]: Peter Lang. S. 61-86.

Häcki Buhofer, Annelies (2002): Der Zusammenhang von Dialektbewusstsein und Charakteristik der gesprochenen Sprache. In: Wiesinger, Peter (Hg.): Akten des X. Internationalen Germanistenkongresses Wien 2000 ,Zeitenwende - Die Germanistik auf dem Weg vom 20. ins 21. Jahrhundert“. Bd. 3: Aufgaben einer zukünftigen Sprachgeschichtsforschung - Gesprochene Sprache in regionaler und sozialer Differenzierung - Sprache in der Öffentlichkeit. Bern [u. a.]: Peter Lang. S. 211-216.

Haas, Walter (1983): Dialekt als Sprache literarischer Werke. In: Besch, Werner [u. a.](Hg.), S. $1637-1651$.

Hasselberg, Joachim/Wegera, Klaus-Peter (1976): Hessisch. Düsseldorf: Schwann (Dialekt/ Hochdeutsch - kontrastiv. Heft 1).

Herrgen, Joachim (1986): Koronalisierung und Hyperkorrektion. Das palatale Allophon des /CH/-Phonems und seine Variation im Westmitteldeutschen. Stuttgart: Steiner (Mainzer Studien zur Sprach- und Volksforschung 9). 
Herrgen, Joachim (2006): Sprachgeographie und Optimalitätstheoric. Am Beispiel der t-Tilgung in Auslaut-Clustern des Deutschen. In: Zeitschrift für Dialektologie und Linguistik 72/2005, S. 277-317.

Herrgen, Joachim/Lenz, Alexandra N. (2003): Digitale Dialektologie. Online-Publikation des Wenker-Atlasses im Internet. In: Marburger UniJournal 14, S. 43-48.

Herrgen, Joachim/Lenz, Alexandra N./Pennay, Mark (2003): The Research Institute for German Language - Deutscher Sprachatlas (Marburg). In: Dialektologia et Geolinguistica 11, S. 94-109.

Herrgen, Joachim/Schmidt, Jürgen Erich (in Vorb.): Sprachdynamik. Eine Einführung in die moderne Regionalsprachenforschung. Berlin: E. Schmidt (Grundlagen der Germanistik).

Hommer, Emil (1910): Studien zur Dialektgeographie des Westerwaldes. Diss. Univ. Marburg.

Huesmann, Anette (1998): Zwischen Dialekt und Standard. Empirische Untersuchung zur Soziolinguistik des Varietätenspektrums im Deutschen. Tübingen: Niemeyer (Reihe Germanistische Linguistik 199).

Kehrein, Roland (2006): Regional accent in the German language area. How dialectally do German police answer emergeny calls? In: Hinskens, Frans (Hg.): Language variation. European perspectives. Amsterdam/Philadelphia: Benjamins. S. 83-96.

Kehrein, Roland/Lameli, Alfred/Nickel, Jost (2005): Möglichkeiten der computergestützten Regionalsprachenforschung am Beispiel des Digitalen Wenker-Atlas (DiWA). In: Braungart, Georg [u. a.] (Hg.): Jahrbuch für Computerphilologie - online 7.

Klein, Eva/Mattheier, Klaus J/Mickartz, Heinz (1978): Rheinisch. Düsseldorf: Schwann (Dialekt/Hochsprache - kontrastiv. Heft 6).

Koch, Peter/Oesterreicher, Wulf (1985): Sprache der Nähe - Sprache der Distanz. Mündlichkeit und Schriftlichkeit im Spannungsfeld von Sprachtheorie und Sprachgeschichte. In: Romanistisches Jahrbuch 36, S. 15-43.

Koch, Peter/Oesterreicher, Wulf (1994): Schriftlichkeit und Sprache. In: Günther, Hartmut/ Ludwig, Otto (Hg.): Schrift und Schriftlichkeit. Ein interdisziplinäres Handbuch internationaler Forschung. 1. Halbbd. Berlin/New York: de Gruyter (Handbücher zur Sprach- und Kommunikationswissenschaft 10.1). S. 587-604.

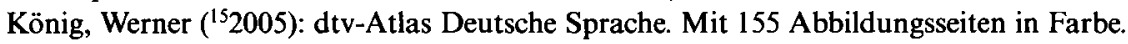
Grafiker Hans-Joachim Paul. München: dtv.

Labov, William (1972): Sociolinguistic Patterns. Philadelphia: University of Pennsylvania Press.

Leirbukt, Oddleif (1997): Untersuchungen zum bekommen-Passiv im heutigen Deutsch. Tübingen: Niemeyer (Reihe Germanistische Linguistik 177).

Lemnitzer, Lothar/Zinsmeister, Heike (2006): Korpuslinguistik. Eine Einführung. Tübingen: Narr (narr studienbücher).

Lenz, Alexandra N. (2003): Struktur und Dynamik des Substandards. Eine Studie zum Westmitteldeutschen (Wittlich/Eifel). Stuttgart: Steiner (Zeitschrift für Dialektologie und Linguistik. Beihefte 125).

Lenz, Alexandra N. (2004): Zur Interpretation des Intendierten Ortsdialekts. In: Lenz, Alexandra N./Radtke, Edgar/Zwickl, Simone (Hg.): Variation im Raum. Variation and Space. Frankfurt/Main: Peter Lang (VarioLingua 20). S. 113-131.

Lenz, Alexandra N. (2005): Hyperdialektalismen und Hyperkorrektionen - Indizien für Varietätengrenzen. In: Lenz, Alexandra N./Mattheier, Klaus J. (Hg.): Varietäten Theorie und Empirie. Frankfurt/M. [u. a.]: Peter Lang (VarioLingua 23). S. 75-95.

Lenz, Alexandra N. (2007a): Zur Grammatikalisierung von geben im Deutschen und Lëtzebuergeschen. In: Zeitschrift für Germanistische Linguistik. 
Lenz, Alexandra N. (2007b): Ergebnisse eines Sprachproduktionsexperiments zum Rezipientenpassiv. In: Patocka, Franz/Seiler, Guido (Hg.): Dialektale Morphologie, dialektale Syntax. Wien: Edition Präsens. [im Druck].

Ludwig, Johannes (1906): Lautlehre der moselfränkischen Mundart von Sehlem (Reg.-Bez. Trier). Diss. Freiburg i. Br.

Lüdtke, Hartmut (2005): Beobachtung/Observation. In: Ammon, Ulrich [u.a.] (Hg.), S. 1033-1051.

Macha, Jürgen (1991): Der flexible Sprecher. Untersuchungen zu Sprache und Sprachbewußtsein rheinischer Handwerksmeister. Köln [u. a.]: Böhlau.

Mattheier, Klaus J. (1979): Sprachvariation und Sprachwandel. Untersuchungen zur Struktur und Entwicklung von Interferenzprozessen zwischen Dialekt und Hochsprache in einer ländlichen Sprachgemeinschaft des Rheinlandes. Habilitationsschrift. Universität Bonn.

McEnery, Tony/Wilson, Andrew (1996): Corpus Linguistics. Edinburgh: University Press.

Mihm, Arend (2004): Zur Geschichte der Auslautverhärtung und ihrer Erforschung. In: Sprachwissenschaft 29, S. 133-206.

Niebaum, Hermann (1977): Westfälisch. Düsseldorf: Schwann (Dialekt/Hochdeutsch kontrastiv. Heft 5).

MRhSA = Bellmann/Herrgen/Schmidt (1994-2002).

Pfeffer, Jay Alan/Lohnes, Walter F. W. (Hg.) (1984): Grunddeutsch. Texte zur gesprochenen deutschen Gegenwartssprache. Überregionale Umgangssprache aus der Bundesrepublik Deutschland, der Deutschen Demokratischen Republik, Österreich und der Schweiz. Textkorpora 1. Einführungs- und Registerband. Tübingen: Niemeyer (Phonai 28).

Rabanus, Stefan (2005): Dialektwandel im 20. Jahrhundert: Verbalplural in Südwestdeutschland. In: Eggers/Schmidt/Stellmacher (Hg.), S. 267-290.

Richter, Helmut (2005): Transkriptionssysteme/Transcription Systems. In: Ammon, Ulrich [u. a.] (Hg.), S. 1126-1134.

Schädlich, Hans-Joachim/Große, Rudolf (1961): Tonbandaufnahme der deutschen Mundarten in der Deutschen Demokratischen Republik. In: Forschungen und Fortschritte 35, S. 358-363.

Schirmunski, Viktor M. (1962): Deutsche Mundartkunde. Vergleichende Laut- und Formenlehre der deutschen Mundarten. Berlin (Veröffentlichungen des Instituts für deutsche Sprache und Literatur 25).

Schlobinski, Peter (1996): Empirische Sprachwissenschaft. Opladen: Westdeutscher Verlag (WV studium 174).

Schmidt, Jürgen Erich (1998): Moderne Dialektologie und regionale Sprachgeschichte. In: Besch, Werner/Solms, Hans Joachim (Hg.): Regionale Sprachgeschichte. Berlin [u. a.] (Zeitschrift für deutsche Philologie. Sonderheft. 117). S. 163-179.

Schmidt, Jürgen Erich (2005): Sprachdynamik. In: Eggers/Schmidt/Stellmacher (Hg.), S. $15-44$.

Schmidt, Jürgen Erich/Herrgen, Joachim (2001 ff.): Digitaler Wenker-Atlas (DiWA). Bearb. von Alfred Lameli, Alexandra N. Lenz, Jost Nickel und Roland Kehrein, Karl-Heinz Müller, Stefan Rabanus. Erste vollständige Ausgabe von Georg Wenkers „Sprachatlas des Deutschen Reichs“. 1888-1923 handgezeichnet von Emil Maurmann, Georg Wenker und Ferdinand Wrede. Marburg: Forschungsinstitut für deutsche Sprache „Deutscher Sprachatlas“. [http://www.diwa.info]

Schwitalla, Johannes/Wegstein, Werner (Hg.) (2005): Korpuslinguistik deutsch: synchron diachron - kontrastiv. Würzburger Kolloquium 2003. Tübingen: Niemeyer.

Siebenhaar, Beat (2003): Sprachgeographische Aspekte der Morphologie und Verschriftung in schweizerdeutschen Chats. In: Linguistik online 15, S. 125-139. 
Siebenhaar, Beat (2005a): Die dialektale Verankerung regionaler Chats in der deutschsprachigen Schweiz. In: Eggers/Schmidt/Stellmacher (Hg.), S. 691-717.

Siebenhaar, Beat (2005b): Varietätenwahl und Code-Switching in Deutschschweizer Chatkanälen. Quantitative und qualitative Analysen. Networx 43 [http://www.mediensprache.net/de/networx/networx_43.pdf].

Siever, Torsten [u.a.] (Hg.) (2005): Websprache.net. Sprache und Kommunikation im Internet. Berlin [u. a.]: de Gruyter (Linguistik. Impulse \& Tendenzen 10).

Skiba, Romuald (2005): Computeranalyse/Computer Analysis. In: Ammon, Ulrich [u. a.] (Hg.), S. 1187-1197.

Spiekermann, Helmut (2005): Regionale Standardisierung, nationale Destandardisierung. In: Eichinger, Ludwig M./Kallmeyer, Werner (Hg.): Standardvariation - Wie viel Variation verträgt die deutsche Sprache? Berlin/New York: Walter de Gruyter (IDSJahrbuch 2004). S. 100-125.

Spolsky, Bernard (2005): Tests/Test. In: Ammon, Ulrich [u. a.] (Hg.), S. 1085-1095.

Stellmacher, Dieter (1981): Niedersächsisch. Düsseldorf: Schwann (Dialekt/Hochsprache kontrastiv. Heft 8).

Stickel, Gerhard (1999): Zur Sprachbefindlichkeit der Deutschen: Erste Ergebnisse einer Repräsentativumfrage. In: Stickel, Gerhard (Hg.): Sprache - Sprachwissenschaft Öffentlichkeit. Berlin/New York: de Gruyter (Jahrbuch 1998 des Instituts für deutsche Sprache). S. 16-44.

Storrer, Angelika (2000): Schriftverkehr auf der Datenautobahn: Besonderheiten der schriftlichen Kommunikation im Internet. In: Voß, G. Günter [u. a.] (Hg.): Neue Medien im Alltag: Begriffsbestimmungen eines interdisziplinären Forschungsfeldes. Opladen: Leske + Budrich. S. 151-176.

Storrer, Angelika (2005): Online-Corpora zur linguistischen Analyse der deutschen Gegenwartssprache. In: Zeitschrift für Germanistische Linguistik 33, S. 145-150.

Thun, Harald (2005): Variation im Gespräch zwischen Informant und Explorator. In: Lenz, Alexandra N./Mattheier, Klaus J. (Hg.): Varietäten - Theorie und Empirie. Frankfurt/M. [u. a.]: Peter Lang (VarioLingua 23). S. 97-126.

Tophinke, Doris/Ziegler, Evelyn (2006): Aber bitte im Kontext': Neue Perspektiven in der dialektologischen Einstellungsforschung. In: Gessinger, Joachim/Voeste, Anja (Hg.): Dialekt im Wandel. Perspektiven einer neuen Dialektologie. Duisburg: Gilles und Franke. (OBST 71). S. 203-222.

Vogel, Petra M. (2003): Passiv in deutschsprachigen Chats. Eine Korpusanalyse. In: Linguistik online $15,3 / 03,141-160$.

Wagener, Peter (2002): Gesprochenes Deutsch online. Zur Modernisierung des Deutschen Spracharchivs. In: Zeitschrift für Dialektologie und Linguistik 69, S. 314335.

Wagener, Peter (2005): Korpuslinguistik im Internet. Neue Wege dialektologischer Forschung am Beispiel des virtuellen Deutschen Spracharchivs. In: Eggers/Schmidt/Stellmacher (Hg.), S. 363-373.

Wagener, Peter/Bausch, Karl-Heinz (1997): Tonaufnahmen des gesprochenen Deutsch. Dokumentation der Bestände von sprachwissenschaftlichen Forschungsprojekten und Archiven. Tübingen: Niemeyer.

Wegera, Klaus-Peter (2000): Gen, oder wie Herr Gottsched will, chen. Zur Geschichte eines Diminutivsuffixes. In: Habermann, Mechthild [u. a.] (Hg.): Wortschatz und Orthographie in Geschichte und Gegenwart. Festschrift für Horst Haider Munske zum 65. Geburtstag. Tübingen: Niemeyer. S. 43-58.

Werlen, Iwar (2000): Variation im gesprochenen Hochdeutschen in der deutschen Schweizam Beispiel der Nachrichten von Radio DRS 1 und Radio DRS 3. In: Häcki Buho- 
fer, Annelies (Hg.): Vom Umgang mit sprachlicher Variation. Soziolinguistik, Dialektologie, Methoden und Wissenschaftsgeschichte. Festschrift für Heinrich Löffler zum 60. Geburtstag. Tübingen, Basel: Francke (Basler Studien zur deutschen Sprache und Literatur 80). S. 311-327.

Wittlicher Wörterbuch ( $\left.{ }^{2} 1984\right)$ : Zusammengestellt von Herrn Georg Fischer. Herausgegeben von Buch- und Offsetdruck, Nels. Wittlich.

Wodak, Ruth (1982): Erhebung von Sprachdaten in natürlicher und simuliert-natürlicher Sprechsituation. In: Besch, Werner [u. a.] (Hg.), S. 539-544.

Wrede, Ferdinand (1895): Über richtige Interpretation der Sprachatlaskarten. Vortrag gehalten in der germanistischen Section der 43. Versammlung deutscher Philologen und Schulmänner zu Köln am 26. September 1895. In: Wenker, Georg/Wrede, Ferdinand: Der Sprachatlas des Deutschen Reichs. Dichtung und Wahrheit. Marburg: Elwert. S. 31-52.

Ziegler, Evelyn (2005): Die Bedeutung von Interaktionsstatus und Interaktionsmodus für die Dialekt-Standard-Variation in der Chatkommunikation. In: Eggers/Schmidt/ Stellmacher (Hg.), S. 719-745.

Ziegler, Evelyn (2006): Identitätskonstruktion und Beziehungsarbeit in bayerischen Schülerzetteln. In: Dürscheid, Christa/ Spitzmüller, Jürgen (Hg.): Perspektiven der Jugendsprachforschung/Trends and Developments in Youth Language Research. Frankfurt/M. [u.a.]: Peter Lang. S. 161-179.

Zifonun, Gisela [u.a.] (1997): Grammatik der deutschen Sprache. 3 Bde. Berlin/New York: de Gruyter (Schriften des Instituts für Deutsche Sprache 7.1-7.3).

Zwirner, Eberhard (1956): Lautdenkmal der deutschen Sprache. In: Zeitschrift für Phonetik und Allgemeine Sprachwissenschaft 9, S. 3-13.

Zwirner, Eberhard (1958): Vorwort. In: Zwirner/Bethge, S. 5-9.

Zwirner, Eberhard/Bethge, Wolfgang (1958): Erläuterungen zu den Texten. Mit 2 Tafeln. Göttingen: Vandenhoeck \& Ruprecht (Lautbibliothek der deutschen Mundarten 1). 